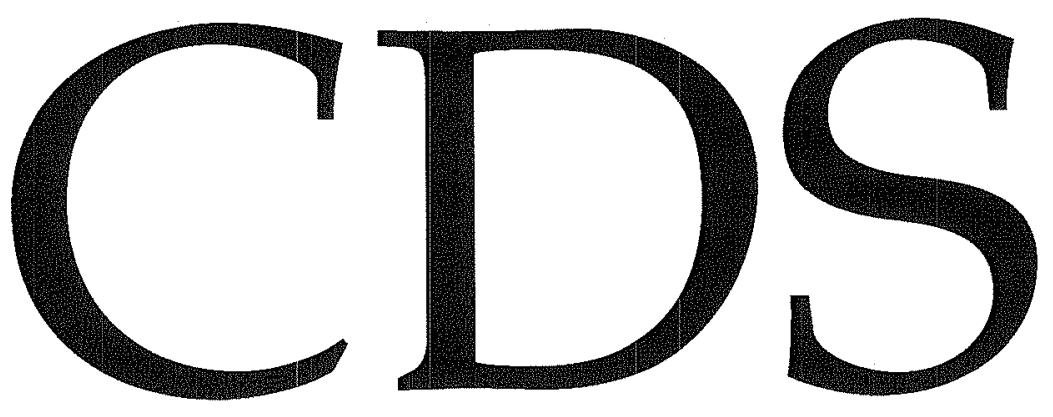

TECHNICAL MEMORANDUM NO. CIT-CDS 93-016

August 30, 1993

"Controller Design with Real Parametric Uncertainty"

Peter M. Young

Control and Dynamical Systems

California Institute of Technology

Pasadena, CA 91125 


\title{
Controller Design with Real Parametric Uncertainty
}

\author{
Peter M. Young \\ Electrical Engineering, 116-81 \\ California Institute of Technology \\ Pasadena, CA 91125, U.S.A.
}

\begin{abstract}
A number of techniques have been developed in recent years for the analysis and design of controllers which are robust with respect to structured complex uncertainty. In particular the complex $\mu$ synthesis procedure has been successfully applied to a number of engineering problems. However the presence of real parametric uncertainty in the problem description substantially complicates matters, so that standard complex $\mu$ synthesis techniques are no longer adequate. In this paper we develop a procedure to tackle the mixed (real and complex) $\mu$ synthesis problem. This procedure involves a " $D, G-K$ iteration" between computing the mixed $\mu$ upper bound and solving an $\mathcal{H}_{\infty}$ optimal control problem, and has guaranteed convergence to a local minimum of the (nonconvex) problem. The procedure has been implemented in software, and several controller designs are compared with the corresponding complex $\mu$ synthesis designs.
\end{abstract}

\section{Introduction}

Many control engineering problems motivate the consideration of robustness problems involving both real and complex uncertainties. These mixed uncertainty descriptions offer the control engineer the possibility of more accurately modeling given physical systems, and hence using less conservative analysis tools, to produce control system designs with enhanced performance. The price to be paid for this added sophistication to the analysis and design procedure is an increase in the complexity of the associated mathematical problems to be solved. A number of practical analysis tools have been developed for mixed problems, and computational software is now available for the general mixed $\mu$ analysis problem [YND92, $\mathrm{BDG}^{+} 91$ ]. In this paper we will be concerned with the general mixed $\mu$ synthesis problem.

The problem of synthesising a controller which is (optimally) robust to structured mixed uncertainty is very difficult, since the associated optimization problem is not convex. Furthermore it seems intuitively clear that the synthesis problem is at least as hard as the analysis problem, which is known to be NP hard [RP, BYDM]. Some exact solutions have been presented for special cases of the synthesis problem (see [RM92] for example, which reduces the rank one $\mu$ synthesis problem to a convex optimization problem), but these are all cases for which the analysis problem also simplifies considerably. As yet there is no globally optimal solution to the general synthesis problem (even in the purely complex case), and no indication that one will be forthcoming in the foreseeable future.

Nevertheless the (complex) $\mu$-synthesis procedure first outlined in [Doy85] has been successfully applied to a large number of engineering problems (see [BYD92] for example). This procedure 
involves a " $D-K$ iteration" between computing the $\mu$ upper bound, and solving for an $H_{\infty}$ (sub) optimal controller (both of which are convex problems). This procedure, which was developed for $\mu$ problems involving only complex blocks, does not guarantee to find the globally $\mu$-optimal controller (since the problem is not jointly convex in $D$ and $K$ ), but has often been found to work well in practice. The approach taken here to the mixed $\mu$ synthesis problem is to extend the above procedure to the mixed case, by exploiting some new analysis tools recently developed for the mixed $\mu$ upper bound. In this way a " $D, G-K$ iteration" procedure is developed, which finds a controller that stabilizes the nominal system, and attempts to minimize the peak value (across frequency) of mixed $\mu$.

Prior to tackling the mixed $\mu$ synthesis problem, we need to develop some State Space factorization machinery, and this is the subject of section 3. A number of factorizations are presented, which are required in the mixed $\mu$ synthesis procedure to essentially produce stable system realizations of the scaling matrices. State Space formulae are presented for all the required factorizations. This machinery is then used in section 4 to develop the " $D, G-K$ iteration" procedure for mixed $\mu$ synthesis. The procedure utilizes a combination of $\mathcal{H}_{\infty}$ optimal control, mixed $\mu$ upper bound computation, and the above factorization machinery to yield an algorithm for mixed $\mu$ synthesis. The algorithm has guaranteed convergence to a local minimum of the problem (as in the complex case). Full details of the procedure, including appropriate routines for fitting the scaling matrices, are given in section 4 . This procedure has been implemented in software, and in section 5 the procedure is applied to several example problems involving real parametric uncertainty. It is seen that the new procedure is able to exploit the phase information in the real uncertainties, to produce a controller with a lower value for mixed $\mu$ (and hence better robust performance) than one could obtain with standard complex $\mu$ synthesis techniques.

\section{Notation and Definitions}

The notation used here is fairly standard, and is taken from [You93]. For any square complex matrix $M$ we denote the transpose by $M^{T}$, and the complex conjugate transpose by $M^{*}$. The largest singular value and the structured singular value are denoted by $\bar{\sigma}(M)$ and $\mu_{\mathcal{K}}(M)$ respectively. For a Hermitian matrix $M$, then $\bar{\lambda}(M)$ denotes the largest (real) eigenvalue. We denote the $k \times k$ identity matrix and zero matrix by $I_{k}$ and $O_{k}$ respectively. Occasionally we will drop the subscripts from $I$ and 0 , whence they denote identity and zero matrices respectively, of the appropriate size.

We define $\mathcal{R M}$ to be the space of real-rational proper transfer matrices. For a transfer matrix $P \in \mathcal{R} \mathcal{M}$, then we denote its State Space representation by

$$
P=\left[\begin{array}{l|l}
A & B \\
\hline C & D
\end{array}\right] \doteq C(s I-A)^{-1} B+D
$$

and we define $P^{\sim}(s) \doteq P^{T}(-s)$. We define $\mathcal{R} \mathcal{L}_{\infty}$ to be the subspace of $\mathcal{R} \mathcal{M}$ with elements analytic on $\operatorname{Re}(s)=0$, and $\mathcal{R} \mathcal{H}_{\infty}$ to be the subspace of $\mathcal{R M}$ with elements analytic in $\operatorname{Re}(s) \geq 0$. Given a transfer matrix $P \in \mathcal{R} \mathcal{H}_{\infty}$, then we denote its infinity norm by

$$
\|P\|_{\infty} \doteq \sup _{\omega \in \mathbb{R}} \bar{\sigma}(P(\mathrm{j} \omega))
$$

The definition of $\mu$ is dependent upon the underlying block structure of the uncertainties, which is defined as follows. Suppose we have a matrix $M \in \mathbb{C}^{n \times n}$ and three non-negative integers $m_{r}, m_{c}$, 
and $m_{C}$ (with $m:=m_{r}+m_{c}+m_{C} \leq n$ ) which specify the number of uncertainty blocks of each type. Then the block structure $\mathcal{K}\left(m_{r}, m_{c}, m_{C}\right)$ is an $m$-tuple of positive integers

$$
\mathcal{K}=\left(k_{1}, \ldots, k_{m_{r}}, k_{m_{r}+1}, \ldots, k_{m_{r}+m_{c}}, k_{m_{r}+m_{c}+1}, \ldots, k_{m}\right)
$$

This $m$-tuple specifies the dimensions of the perturbation blocks, and we require $\sum_{i=1}^{m} k_{i}=n$ in order that these dimensions are compatible with $M$. This determines the set of allowable perturbations, namely define

$$
\begin{aligned}
& X_{\mathcal{K}}=\left\{\Delta=\text { block diag }\left(\delta_{1}^{r} I_{k_{1}}, \ldots, \delta_{m_{r}}^{r} I_{k_{m_{r}}}, \delta_{1}^{c} I_{k_{m_{r}+1}}, \ldots, \delta_{m_{c}}^{c} I_{k_{m_{r}+m_{c}}}, \Delta_{1}^{C}, \ldots, \Delta_{m_{C}}^{C}\right):\right. \\
& \left.\delta_{i}^{r} \in \mathbb{R}, \delta_{i}^{c} \in \mathbb{C}, \Delta_{i}^{C} \in \mathbb{C}^{k_{m_{r}+m_{c}+i} \times k_{m_{r}+m_{c}+i}}\right\}
\end{aligned}
$$

Note that $X_{\mathcal{K}} \subset \mathbb{C}^{n \times n}$ and that this block structure is sufficiently general to allow for (any combination of) repeated real scalars, repeated complex scalars, and full complex blocks. The purely complex case corresponds to $m_{r}=0$, and the purely real case to $m_{c}=m_{C}=0$.

Note also that all the results which follow are easily generalized to the case where the full complex blocks need not be square, and the blocks may come in any order. We make these restrictions in (4) purely for notational convenience.

Definition 1 ([Doy82]) The structured singular value, $\mu_{\mathcal{K}}(M)$, of a matrix $M \in \mathbb{C}^{n \times n}$ with respect to a block structure $\mathcal{K}\left(m_{r}, m_{c}, m_{C}\right)$ is defined as

$$
\mu_{\mathcal{K}}(M)=\left(\min _{\Delta \in X_{\mathcal{K}}}\{\bar{\sigma}(\Delta): \operatorname{det}(I-\Delta M)=0\}\right)^{-1}
$$

with $\mu_{\mathcal{K}}(M)=0$ if no $\Delta \in X_{\mathcal{K}}$ solves $\operatorname{det}(I-\Delta M)=0$.

In order to develop the relevant theory we define the following sets of block diagonal scaling matrices (which, like $\mu$ itself, are dependent on the underlying block structure).

$$
\begin{gathered}
\mathcal{D}_{\mathcal{K}}=\left\{\operatorname{block} \operatorname{diag}\left(D_{1}, \ldots, D_{m_{r}+m_{c}}, d_{1} I_{k_{m_{r}+m_{c}+1}}, \ldots, d_{m_{C}} I_{k_{m}}\right):\right. \\
\left.D_{i} \in \mathbb{C}^{k_{i} \times k_{i}}, \operatorname{det}\left(D_{i}\right) \neq 0, d_{i} \in \mathbb{C}, d_{i} \neq 0\right\} \\
\mathcal{G}_{\mathcal{K}}=\left\{\operatorname{block} \operatorname{diag}\left(G_{1}, \ldots, G_{m_{r}}, O_{k_{m_{r}+1}}, \ldots, O_{k_{m}}\right): G_{i}=G_{i}^{*} \in \mathbb{C}^{k_{i} \times k_{i}}\right\}
\end{gathered}
$$

\section{State Space Factorization Theory}

Prior to considering the mixed $\mu$ synthesis problem we need to develop some State Space factorization machinery. We will develop State Space formulae for specific coprime, inner-outer, and spectral factorizations that will be needed for the mixed $\mu$ synthesis problem. These factorizations are extensions of standard results (see [ZDG] for example), and as such are of some interest in their own right.

\subsection{Basic Concepts}

We begin with a brief review of some basic concepts about transfer matrices. For a more detailed exposition on this subject see [ZDG, Fra87]. 
Definition 2 Suppose we have a transfer matrix $\mathbf{D} \in \mathcal{R} \mathcal{M}$. Then $\mathbf{D}$ is said to be inner if $\mathbf{D} \in$ $\mathcal{R} \mathcal{H}_{\infty}$ and $\mathbf{D}^{\sim} \mathbf{D}=I$, and co-inner if $\mathbf{D} \in \mathcal{R} \mathcal{H}_{\infty}$ and $\mathrm{DD}^{\sim}=I$.

If we are interested in square transfer matrices, then these notions can be simplified somewhat.

Lemma 1 Suppose we have a square transfer matrix $\mathbf{D} \in \mathcal{R} \mathcal{M}$. Then the following conditions are equivalent:

1. $\mathrm{D}^{\sim} \mathrm{D}=I$.

2. $\mathrm{DD}^{\sim}=I$.

3. $\mathrm{D}$ is invertible and $\mathrm{D}^{-1 \sim} \mathrm{D}^{-1}=I$.

4. $\mathrm{D}$ is invertible and $\mathrm{D}^{-1} \mathrm{D}^{-1 \sim}=I$.

Proof: Follows from straightforward manipulations.

Lemma 2 Suppose we have a square transfer matrix $\mathbf{D} \in \mathcal{R} \mathcal{M}$. Then $\mathbf{D}$ is inner if and only if $\mathbf{D}$ is co-inner.

Proof: Apply lemma 1 to $\mathbf{D} \in \mathcal{R} \mathcal{H}_{\infty}$.

A closely related concept is that of an all pass transfer matrix.

Definition 3 Given a square transfer matrix $\mathrm{D} \in \mathcal{R} \mathcal{M}$, then $\mathrm{D}$ is said to be all pass if $\mathrm{D} \in \mathcal{R} \mathcal{L}_{\infty}$ and $\mathbf{D} \sim \mathbf{D}=I$.

Note from lemma 1 that we could equivalently require $\mathbf{D} \in \mathcal{R} \mathcal{L}_{\infty}$ and $\mathbf{D D}^{\sim}=I$. For square matrices then an inner (or co-inner) transfer matrix is exactly a stable all pass transfer matrix.

Lemma 3 Suppose we have a square transfer matrix $\mathrm{D} \in \mathcal{R} \mathcal{M}$. Then $\mathrm{D}$ is all pass if and only if $\mathrm{D}$ is invertible and $\mathrm{D}^{-1}$ is all pass.

Proof: $(\longrightarrow)$ Suppose $D$ is all pass, and hence $D \in \mathcal{R} \mathcal{L}_{\infty}$. From lemma 1 we immediately find that $\mathbf{D}$ is invertible and $\mathbf{D}^{-1 \sim} \mathbf{D}^{-1}=I$. But now this implies $\mathbf{D}^{-1}=\mathbf{D}^{\sim}$, and hence $\mathbf{D}^{-1} \in \mathcal{R} \mathcal{L}_{\infty}$, which together with the earlier result implies $\mathbf{D}^{-1}$ is all pass.

$(\longleftarrow)$ Suppose $\mathbf{D}$ is invertible and $\mathbf{D}^{-1}$ is all pass. Applying the above proof we obtain $\mathbf{D}=\left(\mathbf{D}^{-1}\right)^{-1}$ is all pass.

The term all pass is motivated by the fact that at any given frequency the gain matrix of an all pass transfer matrix is unitary, or, in other words, has unity magnitude gain in all directions. To be more explicit, we have the following well known results (stated without proof).

Lemma 4 Suppose $N_{1}, N_{2}$ are all pass transfer matrices, and $\mathbf{D} \in \mathcal{R M}$. Then for any $\omega \in \mathbb{R}$ we have

$$
\bar{\sigma}\left(N_{1}(\mathbf{j} \omega) \mathbf{D}(\mathbf{j} \omega) N_{2}(\mathbf{j} \omega)\right)=\bar{\sigma}(\mathbf{D}(\mathbf{j} \omega))
$$

Lemma 5 Suppose $N_{1}, N_{2}$ are square inner transfer matrices, and $\mathbf{D} \in \mathcal{R} \mathcal{H}_{\infty}$. Then we have $N_{1} \mathbf{D} N_{2} \in \mathcal{R H}_{\infty}$ and

$$
\left\|N_{1} \mathrm{D} N_{2}\right\|_{\infty}=\|\mathrm{D}\|_{\infty}
$$


Thus the maximum singular value frequency response of a system is invariant to an all pass transfer matrix (and hence the $\mathcal{H}_{\infty}$ norm is invariant to a square inner transfer matrix). This invariance is the reason we are interested in these concepts. The $\mu$ upper bound is based on computing the maximum singular value of a scaled matrix, and it will be seen later that the synthesis problem can be tackled by employing a scaled $\mathcal{H}_{\infty}$ norm optimization procedure, which will rely on the above invariance properties.

The remainder of this paper will make use of the following notions. The term stable refers to a system (matrix) with all its poles (eigenvalues) in the open left half-plane, and marginally stable refers to a system (matrix) with all its poles (eigenvalues) in the closed left half-plane. The term antistable refers to a system (matrix) with all its poles (eigenvalues) in the open right half-plane.

Additionally we will make use of some fairly standard notation and results regarding the solution and properties of Riccati equations. We refer the reader to [DGKF89] for a concise review of this area, and [ZDG] for a more detailed exposition.

\subsection{Coprime Factorization}

Now we wish to develop certain transfer matrix factorizations, the first of which is a coprime factorization. Essentially this amounts to splitting a transfer matrix into stable numerator and denominator matrices, without allowing stable common factors between them. We will use this concept to factor out the antistable part of the original transfer matrix.

Definition 4 Suppose we have two transfer matrices $N, M \in \mathcal{R} \mathcal{M}$. Then $N, M$ are said to be right coprime (over $\mathcal{R} \mathcal{H}_{\infty}$ ) if $N, M \in \mathcal{R} \mathcal{H}_{\infty}$, they have the same number of columns, and $\left(\begin{array}{c}M \\ N\end{array}\right)$ is left invertible in $\mathcal{R H}_{\infty}$.

Given a transfer matrix $\mathbf{D} \in \mathcal{R} \mathcal{M}$, then a right coprime factorization of $\mathbf{D}$ (if it exists) consists of two right coprime transfer matrices $N, M$ such that $\mathbf{D}=N M^{-1}$ (implicitly we have that $M$ is square and invertible). The following theorem addresses the existence of such a factorization.

Theorem 1 Suppose we have a transfer matrix $\mathrm{D} \in \mathcal{R} \mathcal{M}$, and (without loss of generality) that a minimal realization for $\mathbf{D}$ is given by

$$
\mathbf{D}=\left[\begin{array}{c|c}
A & B \\
\hline C & D
\end{array}\right]=\left[\begin{array}{cc|c}
A_{1} & 0 & B_{1} \\
0 & A_{2} & B_{2} \\
\hline C_{1} & C_{2} & D
\end{array}\right]
$$

where $A_{1}$ is marginally stable and $A_{2}$ is antistable. Now defining

$$
H=\left(\begin{array}{cc}
A_{2} & B_{2} B_{2}^{T} \\
0 & -A_{2}^{T}
\end{array}\right)
$$

we have that $H \in \operatorname{dom}($ Ric), and so we can define

$$
\begin{gathered}
X=\operatorname{Ric}(H) \leq 0 \\
N=\left[\begin{array}{cc|c}
A_{1} & B_{1} B_{2}^{T} X & B_{1} \\
0 & A_{2}+B_{2} B_{2}^{T} X & B_{2} \\
\hline C_{1} & C_{2}+D B_{2}^{T} X & D
\end{array}\right]
\end{gathered}
$$




$$
M=\left[\begin{array}{c|c}
A_{2}+B_{2} B_{2}^{T} X & B_{2} \\
\hline B_{2}^{T} X & I
\end{array}\right]
$$

Then we have that $\mathbf{D}=N M^{-1}$, with $N$ marginally stable, $M$ inner, and (12) a minimal realization of $M$. Furthermore if $\mathbf{D} \in \mathcal{R} \mathcal{L}_{\infty}$ then $N \in \mathcal{R} \mathcal{H}_{\infty}$, and $N, M$ are right coprime over $\mathcal{R} \mathcal{H}_{\infty}$.

Proof: Note first that $H$ clearly has no imaginary axis eigenvalues since $A_{2}$ is antistable. Now our assumption of a minimal realization for $\mathrm{D}$ implies that $\left(A_{2}, B_{2}\right)$ is controllable, and hence $\left(A_{2}, B_{2} B_{2}^{T}\right)$ is controllable. Since $B_{2} B_{2}^{T}$ is positive semidefinite, these facts imply that $H \in$ $\operatorname{dom}(\operatorname{Ric})$, so $X=\operatorname{Ric}(H)$ is well defined, and is a real symmetric matrix satisfying the Riccati equation

$$
A_{2}^{T} X+X A_{2}+X B_{2} B_{2}^{T} X=0
$$

with $A_{2}+B_{2} B_{2}^{T} X$ stable (see section 7.2 in [Fra87]). Rewriting this we obtain

$$
\left(A_{2}+B_{2} B_{2}^{T} X\right)^{T} X+X\left(A_{2}+B_{2} B_{2}^{T} X\right)-X B_{2} B_{2}^{T} X=0
$$

This is a Lyapunov equation, and so the fact that $A_{2}+B_{2} B_{2}^{T} X$ is stable and we have $-X B_{2} B_{2}^{T} X \leq 0$ implies that $X \leq 0$. By construction we have that $N$ is marginally stable, and $M \in \mathcal{R} \mathcal{H}_{\infty}$ is square and invertible. Now we have

$$
\mathrm{D} M=\left[\begin{array}{ccc|c}
A_{1} & 0 & B_{1} B_{2}^{T} X & B_{1} \\
0 & A_{2} & B_{2} B_{2}^{T} X & B_{2} \\
0 & 0 & A_{2}+B_{2} B_{2}^{T} X & B_{2} \\
\hline C_{1} & C_{2} & D B_{2}^{T} X & D
\end{array}\right]
$$

Applying the state transformation

$$
T=\left(\begin{array}{lll}
I & 0 & 0 \\
0 & I & 0 \\
0 & I & I
\end{array}\right)
$$

we obtain

$$
\mathbf{D} M=\left[\begin{array}{ccc|c}
A_{1} & B_{1} B_{2}^{T} X & B_{1} B_{2}^{T} X & B_{1} \\
0 & A_{2}+B_{2} B_{2}^{T} X & B_{2} B_{2}^{T} X & B_{2} \\
0 & 0 & A_{2} & 0 \\
\hline C_{1} & C_{2}+D B_{2}^{T} X & D B_{2}^{T} X & D
\end{array}\right]=\left[\begin{array}{cc|c}
A_{1} & B_{1} B_{2}^{T} X & B_{1} \\
0 & A_{2}+B_{2} B_{2}^{T} X & B_{2} \\
\hline C_{1} & C_{2}+D B_{2}^{T} X & D
\end{array}\right]=N
$$

and hence $\mathbf{D}=N M^{-1}$. Furthermore note that

$$
\begin{aligned}
M^{\sim} M & =\left[\begin{array}{cc|c}
-A_{2}^{T}-X B_{2} B_{2}^{T} & -X B_{2} B_{2}^{T} X & -X B_{2} \\
0 & A_{2}+B_{2} B_{2}^{T} X & B_{2} \\
\hline B_{2}^{T} & B_{2}^{T} X & I
\end{array}\right] \\
& =\left[\begin{array}{cc|c}
-A_{2}^{T}-X B_{2} B_{2}^{T} & A_{2}^{T} X+X A_{2}+X B_{2} B_{2}^{T} X & 0 \\
0 & A_{2}+B_{2} B_{2}^{T} X & B_{2} \\
\hline B_{2}^{T} & 0 & I
\end{array}\right] \\
& =\left[\begin{array}{cc|c}
-A_{2}^{T}-X B_{2} B_{2}^{T} & 0 & 0 \\
0 & A_{2}+B_{2} B_{2}^{T} X & B_{2} \\
\hline B_{2}^{T} & 0 & I
\end{array}\right]=I
\end{aligned}
$$

(where we made use of the state transformation $T=\left(\begin{array}{cc}I & -X \\ 0 & I\end{array}\right)$ and the Riccati equation (13)) so that $M$ is inner. Now we know that $\left(A_{2}, B_{2}\right)$ is controllable, $A_{2}$ is antistable, and $A_{2}+B_{2} B_{2}^{T} X$ 
is stable. Applying the $\mathrm{PBH}$ tests to (12), and using these three facts, it is easy to show that $\left(A_{2}+B_{2} B_{2}^{T} X, B_{2}\right)$ is controllable and $\left(B_{2}^{T} X, A_{2}+B_{2} B_{2}^{T} X\right)$ is observable, so that (12) is a minimal realization of $M$. Finally we note that if $\mathrm{D} \in \mathcal{R} \mathcal{L}_{\infty}$ then $A_{1}$ is stable and hence $N \in \mathcal{R} \mathcal{H}_{\infty}$. The fact that $N, M$ are then right coprime over $\mathcal{R} \mathcal{H}_{\infty}$ may be verified by choosing $L$ such that $A+L C$ is stable (which we can do since $(C, A)$ is observable) and checking that

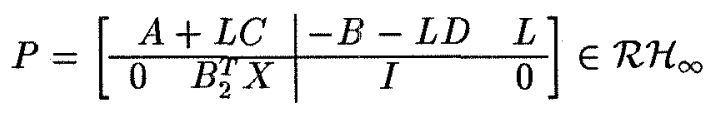

is a left inverse of $\left(\begin{array}{c}M \\ N\end{array}\right)$.

This theorem provides us with State Space formulae to construct a particular factorization, namely one where the denominator matrix $M$ is inner. When $\mathbf{D} \in \mathcal{R} \mathcal{L}_{\infty}$ this is a right coprime factorization, and in fact it is easy to show that such a factorization exists if and only if $D \in \mathcal{R} \mathcal{L}_{\infty}$. Note from the proof that for $\mathbf{D} \notin \mathcal{R} \mathcal{L}_{\infty} A_{1}$ has imaginary axis eigenvalues and so $N \notin \mathcal{R} \mathcal{H}_{\infty}$. However we still have an inner $M$, and a marginally stable $N$, such that $\mathrm{D}=N M^{-1}$, with $P \in \mathcal{R} \mathcal{H}_{\infty}$ (see (14)) a left inverse of $\left(\begin{array}{c}M \\ N\end{array}\right)$.

The existence of a right coprime factorization for $\mathbf{D} \in \mathcal{R} \mathcal{L}_{\infty}$ is well known. What is new here is that the factorization in theorem 1 may be carried out for arbitrary $\mathbf{D} \in \mathcal{R} \mathcal{M}$ and, more importantly, that we only solve a Riccati equation of the same size as $A_{2}$, i.e., the number of antistable poles in $\mathbf{D}$. Thus we only invert out the antistable part of $\mathbf{D}$ and hence obtain a minimal realization for $M$. This offers clear numerical advantages over implementing the standard formulae for coprime factorization (see [ZDG] for example), which would require solving a Riccati equation of the same dimension as $A$, and would result in unobservable modes in the realization for $M$. The numerical advantages of the formulation presented here are particularly important when there is pole symmetry about the imaginary axis, which occurs, for example, when $\mathbf{D}$ is self-adjoint. In that case the Hamiltonian matrix from the standard formulae (see [ZDG, Fra87] for example)

$$
H=\left(\begin{array}{cc}
A & B B^{T} \\
0 & -A^{T}
\end{array}\right)
$$

has every eigenvalue repeated. A standard approach to solving the Riccati equation relies on computing the eigenspaces of the Hamiltonian matrix $H$. It is well known that a non-trivial Jordan form can present severe problems for numerical computation of the eigenvalue decomposition, so that a straightforward implementation of the standard formulae for coprime factorization could run into numerical difficulties on this type of problem.

The analogous definitions and results for left coprime factorizations follow by duality.

Definition 5 Suppose we have two transfer matrices $\tilde{N}, \tilde{M} \in \mathcal{R M}$. Then $\tilde{N}, \tilde{M}$ are said to be left coprime (over $\mathcal{R} \mathcal{H}_{\infty}$ ) if $\tilde{N}, \tilde{M} \in \mathcal{R} \mathcal{H}_{\infty}$, they have the same number of rows, and $(\tilde{M} \tilde{N})$ is right invertible in $\mathcal{R} \mathcal{H}_{\infty}$.

Given a transfer matrix $\mathbf{D} \in \mathcal{R} \mathcal{M}$, then a left coprime factorization of $\mathbf{D}$ (if it exists) consists of two left coprime transfer matrices $\tilde{N}, \tilde{M}$ such that $\mathrm{D}=\tilde{M}^{-1} \tilde{N}$. 
Theorem 2 Suppose we have a transfer matrix $\mathbf{D} \in \mathcal{R} \mathcal{M}$, and (without loss of generality) that a minimal realization for $\mathbf{D}$ is given by

$$
\mathbf{D}=\left[\begin{array}{c|c}
A & B \\
\hline C & D
\end{array}\right]=\left[\begin{array}{cc|c}
A_{1} & 0 & B_{1} \\
0 & A_{2} & B_{2} \\
\hline C_{1} & C_{2} & D
\end{array}\right]
$$

where $A_{1}$ is marginally stable and $A_{2}$ is antistable. Now defining

$$
H=\left(\begin{array}{cc}
A_{2}^{T} & C_{2}^{T} C_{2} \\
0 & -A_{2}
\end{array}\right)
$$

we have that $H \in \operatorname{dom}($ Ric), and so we can define

$$
\begin{gathered}
X=\operatorname{Ric}(H) \leq 0 \\
\tilde{N}=\left[\begin{array}{cc|c}
A_{1} & 0 & B_{1} \\
X C_{2}^{T} C_{1} & A_{2}+X C_{2}^{T} C_{2} & B_{2}+X C_{2}^{T} D \\
\hline C_{1} & D
\end{array}\right] \\
\tilde{M}=\left[\begin{array}{c|c}
A_{2}+X C_{2}^{T} C_{2} & X C_{2}^{T} \\
\hline C_{2} & I
\end{array}\right]
\end{gathered}
$$

Then we have that $\mathbf{D}=\tilde{M}^{-1} \tilde{N}$, with $\tilde{N}$ marginally stable, $\tilde{M}$ inner, and (19) a minimal realization of $\tilde{M}$. Furthermore if $\mathrm{D} \in \mathcal{R} \mathcal{L}_{\infty}$ then $\tilde{N} \in \mathcal{R} \mathcal{H}_{\infty}$, and $\tilde{N}, \tilde{M}$ are left coprime over $\mathcal{R} \mathcal{H}_{\infty}$.

Proof: Apply theorem 1 to $\mathbf{D}^{T}$ and the results follow.

\subsection{Inner-Outer Factorization}

In this section we will make use of the following notions. The term minimum phase refers to a system (matrix) with all its zeros (eigenvalues) in the open left half-plane, and marginally minimum phase refers to a system (matrix) with all its zeros (eigenvalues) in the closed left half-plane. The term anti minimum phase refers to a system (matrix) with all its zeros (eigenvalues) in the open right half-plane.

We now wish to develop a factorization which will factor out the anti minimum phase part of a transfer matrix. It is useful to consider another special type of transfer matrix.

Definition 6 Suppose we have a transfer matrix $\mathrm{D} \in \mathcal{R} \mathcal{M}$. Then $\mathrm{D}$ is outer if $\mathrm{D} \in \mathcal{R} \mathcal{H}_{\infty}$ and $\mathrm{D}$ is right invertible in $\mathcal{R} \mathcal{H}_{\infty}$.

It is easy to show that a square transfer matrix $\mathbf{D} \in \mathcal{R M}$ is outer if and only if $\mathbf{D}, \mathbf{D}^{-1} \in \mathcal{R} \mathcal{H}_{\infty}$. Thus an outer matrix is stable minimum phase, i.e., it has all its poles and zeros in the open left half-plane. Note that in an analogous fashion we can define a matrix $\mathbf{D} \in \mathcal{R M}$ as co-outer if $\mathbf{D} \in \mathcal{R} \mathcal{H}_{\infty}$ and $\mathbf{D}$ is left invertible in $\mathcal{R} \mathcal{H}_{\infty}$. It is clear that for square transfer matrices $\mathbf{D}$ is outer if and only if $\mathbf{D}$ is co-outer.

If we can factor a matrix $\mathbf{D} \in \mathcal{R} \mathcal{M}$ as $\mathbf{D}=\mathbf{D}_{i} \mathbf{D}_{o}$, with $\mathbf{D}_{i}$ inner, and $\mathbf{D}_{o}$ outer, then this is referred to as an inner-outer factorization of $\mathbf{D}$. Clearly we must have $\mathbf{D} \in \mathcal{R} \mathcal{H}_{\infty}$ for such a factorization to exist, and in fact it can be shown that such a factorization exists for all $\mathbf{D} \in \mathcal{R} \mathcal{H}_{\infty}$ (see [ZDG, Fra87]). Here we need only concern ourselves with a special case of this factorization, namely when $\mathbf{D}$ is square and invertible. 
Theorem 3 Suppose $\mathbf{D} \in \mathcal{R} \mathcal{M}$ is square, invertible, and marginally stable. Further suppose (without loss of generality) that we have a minimal realization for $\mathrm{D}^{-1}$

$$
\mathrm{D}^{-1}=\left[\begin{array}{c|c}
A & B \\
\hline C & D
\end{array}\right]=\left[\begin{array}{cc|c}
A_{1} & 0 & B_{1} \\
0 & A_{2} & B_{2} \\
\hline C_{1} & C_{2} & D
\end{array}\right]
$$

with $A_{1}$ marginally stable, and $A_{2}$ anti-stable, so that defining

$$
\begin{aligned}
& \hat{A}=A-B D^{-1} C \\
& \hat{B}=B D^{-1} \\
& \hat{C}=-D^{-1} C \\
& \hat{D}=D^{-1}
\end{aligned}
$$

we have that

$$
\mathrm{D}=\left[\begin{array}{c|c}
\hat{A} & \hat{B} \\
\hline \hat{C} & \hat{D}
\end{array}\right]=\left[\begin{array}{cc|c}
\hat{A} & \hat{B} \\
\hline \hat{C}_{1} & \hat{C}_{2} & \hat{D}
\end{array}\right]
$$

is a minimal realization for $\mathbf{D}$. Now defining

$$
H=\left(\begin{array}{cc}
A_{2} & B_{2} B_{2}^{T} \\
0 & -A_{2}^{T}
\end{array}\right)
$$

we have that $H \in \operatorname{dom}($ Ric), and so we can define

$$
\begin{gathered}
X=\operatorname{Ric}(H) \leq 0 \\
\mathbf{D}_{i}=\left[\begin{array}{c|c}
A_{2}+B_{2} B_{2}^{T} X & B_{2} \\
\hline B_{2}^{T} X & I
\end{array}\right] \\
\mathbf{D}_{o}=\left[\begin{array}{cc|c}
\hat{A} & \hat{B} \\
\hline \hat{C}_{1} & \hat{C}_{2}-B_{2}^{T} X & \hat{D}
\end{array}\right]
\end{gathered}
$$

Then we have that $\mathbf{D}=\mathbf{D}_{i} \mathbf{D}_{o}$ with $\mathbf{D}_{i}$ inner, and (25) a minimal realization for $\mathbf{D}_{i}$, and $\mathbf{D}_{o}$ marginally stable, marginally minimum phase. Furthermore if $\mathbf{D} \in \mathcal{R} \mathcal{H}_{\infty}$ then $\mathbf{D}_{o} \in \mathcal{R} \mathcal{H}_{\infty}$ and if $\mathbf{D}^{-1} \in \mathcal{R} \mathcal{L}_{\infty}$ then $\mathbb{D}_{o}$ is (right) invertible in $\mathcal{R} \mathcal{H}_{\infty}$.

Proof: Apply theorem 1 to factor $\mathrm{D}^{-1}$ as $\mathrm{D}^{-1}=N M^{-1}$ with

$$
\begin{gathered}
N=\left[\begin{array}{cc|c}
A_{1} & B_{1} B_{2}^{T} X & B_{1} \\
0 & A_{2}+B_{2} B_{2}^{T} X & B_{2} \\
\hline C_{1} & C_{2}+D B_{2}^{T} X & D
\end{array}\right] \\
M=\left[\begin{array}{c|c}
A_{2}+B_{2} B_{2}^{T} X & B_{2} \\
\hline \bar{B}_{2}^{T} X & I
\end{array}\right]
\end{gathered}
$$

where $X=\operatorname{Ric}(H) \leq 0$. Note that $N$ is marginally stable, $M$ is inner, and (28) is a minimal realization of $M$. Note also that $N$ is square and invertible (as is $M$ ). Now define $\mathbf{D}_{i}=M$ and $\mathrm{D}_{o}=N^{-1}$, and we have

$$
\mathbf{D}=\left(\mathbf{D}^{-1}\right)^{-1}=\left(N M^{-1}\right)^{-1}=M N^{-1}=\mathbf{D}_{i} \mathbf{D}_{o}
$$


with $\mathbf{D}_{i}$ inner, (25) a minimal realization of $\mathbf{D}_{i}$, and $\mathbf{D}_{o}$ marginally minimum phase (since $\mathbf{D}_{o}^{-1}$ is marginally stable). Now by construction we have

$$
\mathbf{D}_{o}=\left[\begin{array}{cc|c}
A_{1} & B_{1} B_{2}^{T} X & B_{1} \\
0 & A_{2}+B_{2} B_{2}^{T} X & B_{2} \\
\hline C_{1} & C_{2}+D B_{2}^{T} X & D
\end{array}\right]^{-1}=\left[\begin{array}{cc|c} 
& \hat{A} & \hat{B} \\
\hline \hat{C}_{1} & \hat{C}_{2}-B_{2}^{T} X & \hat{D}
\end{array}\right]
$$

so that $\mathbf{D}_{o}$ is marginally stable. Finally note that if $\mathbf{D} \in \mathcal{R} \mathcal{H}_{\infty}$ then $\hat{A}$ is stable so $\mathbf{D}_{o} \in \mathcal{R} \mathcal{H}_{\infty}$, and if $\mathbf{D}^{-1} \in \mathcal{R} \mathcal{L}_{\infty}$ then $N \in \mathcal{R} \mathcal{H}_{\infty}$, so $\mathbf{D}_{o}$ is (right) invertible in $\mathcal{R H}_{\infty}$ (with $\mathbf{D}_{o}^{-1}=N$ ).

In the case that $\mathbf{D} \in \mathcal{R} \mathcal{H}_{\infty}, \mathbf{D}^{-1} \in \mathcal{R} \mathcal{L}_{\infty}$ we have that $\mathbf{D}_{o}$ is outer and hence this is exactly an inner-outer factorization. Note once again that although the inner-outer factorization is well known, we are here only solving a Riccati equation of the same dimension as $A_{1}$, i.e., the anti minimum phase zeros of $D$. Thus by only factoring out the anti minimum phase part of $D$ we obtain a minimal realization for $\mathbf{D}_{i}$. This offers numerical advantages over the standard approach, and similar comments to those made in section 3.2 apply.

Once again we may define a dual version of this factorization, namely if we can factor a matrix $\mathbf{D} \in \mathcal{R} \mathcal{M}$ as $\mathbf{D}=\mathbf{D}_{c o} \mathbf{D}_{c i}$, with $\mathbf{D}_{c i}$ co-inner, and $\mathbf{D}_{c o}$ co-outer, then this is referred to as a coouter-co-inner factorization of $\mathbf{D}$. Again the results follow easily by duality, and we do not include them here

\subsection{Combined Factorizations}

The machinery presented in the preceding subsections enables us to carry out a combined factorization, which splits off the anti-stable poles and anti minimum phase zeros of $\mathbf{D}$ using all pass functions.

Theorem 4 Suppose we have a square invertible transfer matrix $\mathrm{D} \in \mathcal{R} \mathcal{M}$. Then $\mathrm{D}$ may be factored as

$$
\mathbf{D}=\mathbf{D}_{a p a} \mathbf{D}_{a p s} \mathbf{D}_{s m p}
$$

where $\mathbf{D}_{\text {apa }}$ is anti stable all pass, $\mathbf{D}_{\text {aps }}$ is stable all pass, and $\mathbf{D}_{\text {smp }}$ is marginally stable, marginally minimum phase. Furthermore if $\mathbf{D} \in \mathcal{R} \mathcal{L}_{\infty}$ then $\mathbf{D}_{\text {smp }}$ is stable, and if $\mathbf{D}^{-1} \in \mathcal{R} \mathcal{L}_{\infty}$ then $\mathbf{D}_{\text {smp }}$ is minimum phase.

Proof: Apply theorem 2 to obtain

$$
\mathrm{D}=\tilde{M}^{-1} \tilde{N}
$$

with $\tilde{M}$ inner, and $\tilde{N}$ marginally stable. Define $\mathbf{D}_{a p a}=\tilde{M}^{-1}$ and it is easy to check from (19) that $\tilde{M}^{-1}$ is anti-stable. Now apply theorem 3 to obtain

$$
\tilde{N}=\mathrm{D}_{a p s} \mathrm{D}_{s m p}
$$

with $\mathbf{D}_{\text {aps }}$ inner and $\mathbf{D}_{s m p}$ marginally stable, marginally minimum phase. The results now follow.

Note that for $\mathbf{D}, \mathbf{D}^{-1} \in \mathcal{R} \mathcal{L}_{\infty}$ (which will be the case when this result is employed in the mixed $\mu$ synthesis problem) this allows us to factor $\mathbf{D}$ such that

$$
\mathrm{D}=\mathbf{D}_{a p} \mathbf{D}_{s m p}
$$


where $\mathbf{D}_{a p}=\mathbf{D}_{\text {apa }} \mathbf{D}_{\text {aps }}$ is an all pass function, and $\mathbf{D}_{s m p}$ is stable minimum phase. The State Space formulae to perform this factorization are given in theorems 2 and 3 . In order to compute this factorization we need to solve two Riccati equations. Note that these two Riccati equations have dimension equal to the number of anti-stable poles of $\mathbf{D}$, and the number of anti minimum phase zeros of $\mathbf{D}$ respectively.

\subsection{Spectral Factorization}

Finally we consider spectral factorization, which is essentially a transfer matrix equivalent of the square root. We are concerned with a particular form of spectral factorization, that arises from the mixed $\mu$ upper bound.

Theorem 5 Suppose we have a square transfer matrix $G \in \mathcal{R} \mathcal{M}$. Further suppose (without loss of generality) that a minimal realization for $G$ is given by

$$
G=\left[\begin{array}{l|l}
A & B \\
\hline C & D
\end{array}\right]
$$

Now define the symmetric positive definite matrices $R$ and $Q$ as

$$
\begin{aligned}
& R=I+D^{T} D \\
& Q=I+D D^{T}
\end{aligned}
$$

and define the Hamiltonian matrix

$$
H=\left(\begin{array}{cc}
A-B R^{-1} D^{T} C & -B R^{-1} B^{T} \\
-C^{T} Q^{-1} C & -\left(A-B R^{-1} D^{T} C\right)^{T}
\end{array}\right)
$$

Then $H \in \operatorname{dom}($ Ric) so we may define

$$
\begin{gathered}
X=\operatorname{Ric}(H)>0 \\
G_{h}=\left[\begin{array}{c|c}
A-B R^{-1} D^{T} C-B R^{-1} B^{T} X & B R^{-\frac{1}{2}} \\
\hline-R^{-1}\left(D^{T} C+B^{T} X\right) & R^{-\frac{1}{2}}
\end{array}\right]
\end{gathered}
$$

Then we have that $G_{h} \in \mathcal{R} \mathcal{H}_{\infty}$ and

$$
\left(I+G^{\sim} G\right)^{-1}=G_{h} G_{h}^{\sim}
$$

with $G G_{h} \in \mathcal{R} \mathcal{H}_{\infty}$ given by

$$
G G_{h}=\left[\begin{array}{c|c}
A-B R^{-1} D^{T} C-B R^{-1} B^{T} X & B R^{-\frac{1}{2}} \\
\hline Q^{-1}\left(C-D B^{T} X\right) & D R^{-\frac{1}{2}}
\end{array}\right]
$$

Proof: First we make the definitions

$$
\begin{aligned}
& \hat{A}=A-B R^{-1} D^{T} C \\
& \hat{B}=B R^{-\frac{1}{2}} \\
& \hat{C}=Q^{-\frac{1}{2}} C
\end{aligned}
$$


It is easy to show that $(\hat{A}, \hat{B})$ is controllable and $(\hat{C}, \hat{A})$ is observable. But now we note that $H$ has the form

$$
H=\left(\begin{array}{cc}
\hat{A} & -\hat{B} \hat{B}^{T} \\
-\hat{C}^{T} \hat{C} & -\hat{A}^{T}
\end{array}\right)
$$

and hence we have that $H \in \operatorname{dom}(\operatorname{Ric})$, so that $X=\operatorname{Ric}(H)$ is well defined and $X>0$ (see lemma 3 in [DGKF 89]) satisfies the Riccati equation

$$
\left(A-B R^{-1} D^{T} C\right)^{T} X+X\left(A-B R^{-1} D^{T} C\right)-X B R^{-1} B^{T} X+C^{T} Q^{-1} C=0
$$

with $A-B R^{-1} D^{T} C-B R^{-1} B^{T} X$ stable. Thus by construction $G_{h} \in \mathcal{R} \mathcal{H}_{\infty}$, and $G_{h}$ is square and invertible. Now define

$$
N=G_{h}^{-1}=\left[\begin{array}{c|c}
A & B \\
\hline R^{-\frac{1}{2}}\left(D^{T} C+B^{T} X\right) & R^{\frac{1}{2}}
\end{array}\right]
$$

and we have

$$
\begin{aligned}
N^{\sim} N & =\left[\begin{array}{cc|c}
A & 0 & B \\
-\left(C^{T} D+X B\right) R^{-1}\left(D^{T} C+B^{T} X\right) & -A^{T} & -C^{T} D-X B \\
\hline D^{T} C+B^{T} X & B^{T} & R
\end{array}\right] \\
& =\left[\begin{array}{cc|c}
A & 0 & B \\
-\left(A^{T} X+X A+C^{T} C\right) & -A^{T} & -C^{T} D-X B \\
\hline D^{T} C+B^{T} X & B^{T} & R
\end{array}\right] \\
& =\left[\begin{array}{cc|c}
A & 0 & B \\
-C^{T} C & -A^{T} & -C^{T} D \\
\hline D^{T} C & B^{T} & R
\end{array}\right] \\
& =I+G^{\sim} G
\end{aligned}
$$

Note we have made use of the Riccati equation (39) and the similarity transformation $T=$ $\left(\begin{array}{cc}I & 0 \\ -X & I\end{array}\right)$. Thus we have

$$
\left(I+G^{\sim} G\right)^{-1}=\left(N^{\sim} N\right)^{-1}=N^{-1} N^{-1 \sim}=G_{h} G_{h}^{\sim}
$$

Finally we have that

$$
\begin{aligned}
G G_{h} & =\left[\begin{array}{cc|c}
A-B R^{-1} D^{T} C-B R^{-1} B^{T} X & 0 & B R^{-\frac{1}{2}} \\
-B R^{-1} D^{T} C-B R^{-1} B^{T} X & A & B R^{-\frac{1}{2}} \\
\hline-D R^{-1}\left(D^{T} C+B^{T} X\right) & C & D R^{-\frac{1}{2}}
\end{array}\right] \\
& =\left[\begin{array}{cc|c}
A & 0 \\
-B R^{-1} D^{T} C-B R^{-1} B^{T} X & A-B R^{-1} D^{T} C-B R^{-1} B^{T} X & B R^{-\frac{1}{2}} \\
\hline-D R^{-1}\left(D^{T} C+B^{T} X\right) & C-D R^{-1}\left(D^{T} C+B^{T} X\right) & D R^{-\frac{1}{2}}
\end{array}\right] \\
& =\left[\begin{array}{c|c}
A-B R^{-1} D^{T} C-B R^{-1} B^{T} X & B R^{-\frac{1}{2}} \\
\hline Q^{-1}\left(C-D B^{T} X\right) & D R^{-\frac{1}{2}}
\end{array}\right]
\end{aligned}
$$

(where we have used the similarity transformation $T=\left(\begin{array}{cc}I & I \\ 0 & I\end{array}\right)$ ) and hence $G G_{h} \in \mathcal{R} \mathcal{H}_{\infty}$. 
Essentially $G_{h}$ is a stable realization of $\left(I+G^{\sim} G\right)^{-\frac{1}{2}}$. Note that we do not make any restrictions on the pole/zero locations of $G$ for the existence of this factorization. In particular $G$ may have poles and/or zeros on the imaginary axis, and we still obtain stable realizations for $G_{h}$ and $G G_{h}$. It will be seen later that this degree of generality is required for the mixed $\mu$ synthesis problem.

\section{Controller Synthesis}

We now wish to consider the problem of synthesising a controller to meet a certain robust performance requirement. First we place the problem in a standard framework as shown in figure 1.

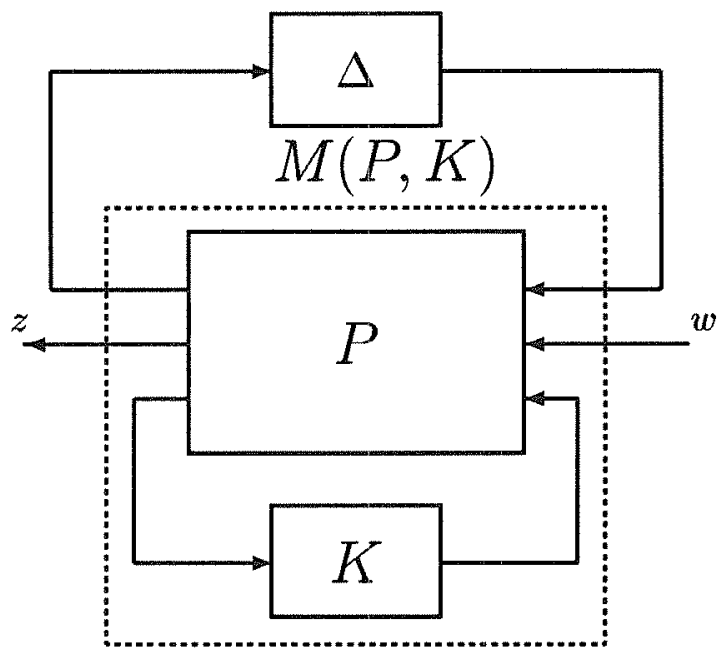

Figure 1: Feedback interconnection for $\mu$ synthesis

The transfer matrix $\mathbf{P}$ is assumed here to contain all the appropriate weights for the problem, as well as the interconnection structure pertaining to the model of the physical plant. Note that we can define the transfer matrix $\mathbf{M}(\mathbf{P}, \mathbf{K})$, by absorbing the controller $\mathbf{K}$ into $\mathbf{P}$ as shown in figure 1 , and so place the problem in the standard robust performance $\mu$ analysis framework (see [PD93]).

We are interested in developing methods to tackle the general $\mu$ synthesis problem, namely that of finding a controller $\mathbf{K}$ achieving

$$
\inf _{\mathbf{K} \in \mathcal{K}_{\mathcal{S}}} \sup _{\omega \in \mathbb{R}} \mu_{\mathcal{K}}(\mathbf{M}(\mathbf{P}, \mathbf{K})(\mathbf{j} \omega))
$$

where $\mathcal{K}_{\mathcal{S}}$ denotes all real rational proper controllers that nominally stabilize $\mathbf{P}$ (i. e., render $\mathbf{M}(\mathbf{P}, \mathbf{K})$ internally stable). The control engineering motivation for this problem is extensive, and may be found elsewhere (see [PD93, YND91, You93] and the references therein), together with the precise mathematical robustness interpretation of (41). Here we merely note that a solution to (41) gives us a controller achieving the best possible robust performance (in an $\mathcal{H}_{\infty}$ norm sense) with respect to a certain perturbation class. Note also that here we are explicitly including real uncertainties in the block structure, so that this synthesis procedure considers parametric uncertainty directly, rather than simply approximating the real uncertainties with complex ones, and applying standard complex $\mu$ synthesis techniques. 


\subsection{Complex $\mu$ Synthesis and " $D$ - $K$ Iteration"}

Prior to tackling the mixed $\mu$ synthesis problem, we first include a very brief review of complex $\mu$ synthesis (where $m_{r}=0$ ). For a more detailed exposition see [Doy85, SD91]. First note that the synthesis problem (41) is not tractable, and so we consider the problem given by replacing complex $\mu$ by its upper bound in (41), namely (see [Doy85])

$$
\inf _{\mathbf{K} \in \mathcal{K}_{\mathcal{S}}} \sup _{\omega \in \mathbb{R}} \inf _{D(\omega) \in \mathcal{D}_{\mathcal{K}}} \bar{\sigma}\left(D(\omega) \mathbf{M}(\mathbf{P}, \mathbf{K})(\mathbf{j} \omega) D^{-1}(\omega)\right)
$$

Note that if we fix $\mathbf{K}$ then the problem of finding $D(\omega)$ is just the standard complex $\mu$ upper bound problem (across frequency) which is a convex problem and can be efficiently solved. If we choose $D(\omega)$ matrices at a set of frequency points (from the $\mu$ upper bound) we can fit a real rational, stable, minimum-phase transfer matrix to them. If we fix this $\mathbf{D}$ transfer matrix then the problem of finding $\mathbf{K}$ reduces to a standard $\mathcal{H}_{\infty}$ problem, as follows. Consider the feedback interconnection

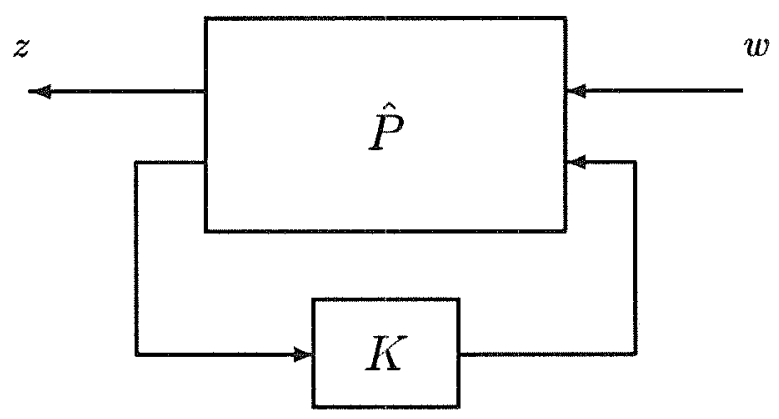

Figure 2: Standard framework for $\mathcal{H}_{\infty}$ optimal control

in figure 2. Assume once again that we have collected all the relevant quantities in the nominal generalized plant $\hat{P}$, and denote by $T_{z w}$ the closed loop transfer function from $w$ to $z$. Then the problem of choosing a real rational proper controller $\mathbf{K}$, so as to render this feedback interconnection internally stable, and minimize $\left\|T_{z w}\right\|_{\infty}$, is the standard $\mathcal{H}_{\infty}$ optimal control problem. This problem is convex and a solution was obtained in [DGKF89], for which commercial software is now available $\left[\mathrm{BPD}^{+}\right.$91].

The above approach leads to the following " $D-K$ iteration" scheme, which attempts to find a complex $\mu$ optimal controller (and hence the best achievable robust performance for problems with structured complex uncertainty):

\section{Procedure 1 ( $D$ - $K$ Iteration)}

1. Find an initial estimate of the scaling matrices $D(\omega)$ pointwise across frequency. One possibility is to use the identity matrix at each point.

2. Find a State Space realization, D, fitting the pointwise scaling matrices $D(\omega)$ with a stable minimum phase system (so that $\mathbf{D}$ and $\mathrm{D}^{-1}$ are stable). Augment this with an identity matrix of the appropriate size so that $\mathrm{D}$ is compatible with $\mathrm{P}$. Construct the State Space system $\mathbf{P}_{\mathbf{D}}=\mathrm{DPD}^{-1}$. 
3. Find the $\mathcal{H}_{\infty}$ optimal controller $\hat{\mathbf{K}}$ minimizing $\left\|\mathbf{M}\left(\mathbf{P}_{\mathbf{D}}, \mathbf{K}\right)\right\|_{\infty}$ over all all stabilizing, proper, real rational controllers $\mathbf{K}$.

4. Find $\hat{D}(\omega)$ solving the minimization problem

$$
\inf _{D(\omega) \in \mathcal{D}_{\mathcal{K}}} \bar{\sigma}\left(D(\omega) \mathbf{M}(\mathbf{P}, \hat{\mathbf{K}})(\mathbf{j} \omega) D^{-1}(\omega)\right)
$$

pointwise across frequency.

5. Compare $\hat{D}(\omega)$ with the previous estimate $D(\omega)$. Stop if they are close, else replace $D(\omega)$ with $\hat{D}(\omega)$ and return to step 2.

This iteration (assuming perfect State Space realizations of $D(\omega)$ ) is monotonically nonincreasing, so that we are guaranteed convergence to a local minimum of the problem. Having converged to such a point the controller $\hat{\mathbf{K}}$ from step 3 is the resulting complex $\mu$ synthesis controller. Note that although the individual problems (the $\mu$ upper bound and $\mathcal{H}_{\infty}$ optimal control) are convex, the joint problem ( $\mu$ optimal control) is not convex (see [Doy85] for a counterexample to convexity). Thus the " $D-K$ iteration" described above is not guaranteed to converge to the global optimum of the problem. However many designs have been performed using this technique in recent years (see [BCD89, BD90] for example), and it has usually been found to work well in practice. There are further subtleties to the above procedure, which we will not go into here (see [SD91, $\mathrm{BDG}^{+} 91$ ] for more details).

This " $D-K$ iteration" scheme is the basis of complex $\mu$ synthesis controller design. The design process consists essentially of the following stages.

\section{Procedure 2 (Complex $\mu$ Synthesis)}

1. Decide on an appropriate interconnection structure to model the system, including the uncertainty structure against which robustness is desired.

2. Choose appropriate weights to reflect the desired performance specifications, and any information known about the uncertainties.

3. Implement the above "D-K iteration". Note that this involves deciding on an appropriate frequency range of interest, and selecting the order of the State Space fit in step 2.

This procedure is by no means mechanical, and a great deal of engineering judgement is still required, particularly in steps 1 and 2. Also, as with any control design technique, the full design process may well involve an iterative application of the above process, together with an evaluation of each resulting controller.

\subsection{Mixed $\mu$ Synthesis}

As in the complex case we note that (41) is not tractable, and so we consider the problem given by replacing $\mu$ by its upper bound in (41). Now for any matrix $M \in \mathbb{C}^{n \times n}$ we have that (see [FTD91, You93])

$$
\mu_{\mathcal{K}}(M) \leq \inf _{D \in \mathcal{D}_{\mathcal{K}}, G \in \mathcal{G}_{\mathcal{K}}} \inf _{\beta \in \mathbb{R}, \beta>0}\left\{\beta: \bar{\sigma}\left(\left(\frac{D M D^{-1}}{\beta}-\mathrm{j} G\right)\left(I+G^{2}\right)^{-\frac{1}{2}}\right) \leq 1\right\}
$$


Note that if we enforce the choice $G=0_{n}$ then we recover the standard complex $\mu$ upper bound. The additional degrees of freedom we have in choosing the $G$ matrix enable us to exploit the phase information we have about the real uncertainties to get a better bound. Note also that this problem may be rearranged into a quasi-convex optimization problem, for which efficient computational software exists (we refer the interested reader to [YND92] for details of the practical computation of the mixed $\mu$ upper bound and associated scaling matrices). This leads us to consider the following synthesis problem.

$$
\inf _{K \in \mathcal{K}_{\mathcal{S}}} \sup _{\omega \in \mathbb{R}} \inf _{D(\omega) \in \mathcal{D}_{\mathcal{K}}, G(\omega) \in G_{\mathcal{K}}} \inf _{\beta(\omega) \in \mathbb{R}, \beta(\omega)>0}\{\beta(\omega): \Gamma \leq 1\}
$$

where

$$
\Gamma=\bar{\sigma}\left(\left(\frac{D(\omega) \mathrm{M}(\mathbf{P}, \mathbf{K})(\mathbf{j} \omega) D^{-1}(\omega)}{\beta(\omega)}-\mathrm{j} G(\omega)\right)\left(I+G^{2}(\omega)\right)^{-\frac{1}{2}}\right)
$$

The basic idea behind our approach to mixed $\mu$ synthesis is evident from these equations $(44,45)$. For fixed $\mathrm{K}$ then the problem of finding $D(\omega), G(\omega), \beta(\omega)$ is just the mixed $\mu$ upper bound problem (across frequency), which is a convex problem and can be efficiently solved. Having chosen $D(\omega), G(\omega), \beta(\omega)$ at a set of frequency points (from the $\mu$ upper bound), we will fit real rational transfer matrices to them, in such a way that the interconnection is stable. For fixed D, G, $\beta$ transfer matrices then the problem of finding $\mathrm{K}$ will be reduced to a standard $\mathcal{H}_{\infty}$ problem which again is convex and can be efficiently solved. This will lead to a " $D, G-K$ iteration" scheme, for the mixed $\mu$ synthesis problem.

Of course we will suffer from the same limitations as the " $D-K$ iteration" procedure for complex $\mu$ synthesis, namely that the joint problem of optimizing $\mathbf{D}, \mathbf{G}$ and $\mathbf{K}$ is not convex. Thus the scheme will not guarantee to find the global optimum of the problem, but rather only a local minimum. Nevertheless we will obtain a procedure which exploits the phase information in the real uncertainties, via the $\mathbf{G}$ scaling matrix, and so has the potential of greatly improving on the present approach, where one simply covers the real uncertainties with complex ones, and applies complex $\mu$ synthesis.

\subsection{1 " $D, G-K$ Iteration"}

Note that the problem statement in equations $(44,45)$ is not quite in the form of a (scaled) singular value minimization, but rather a minimization subject to a (scaled) singular value constraint. In order to proceed we need to exploit some properties of the mixed $\mu$ upper bound.

Theorem 6 Suppose we have matrices $M \in \mathbb{C}^{n \times n}, D \in \mathcal{D}_{\mathcal{K}}, G \in \mathcal{G}_{\mathcal{K}}$ and a real scalar $\beta>0$ such that

$$
\bar{\sigma}\left(\left(\frac{D M D^{-1}}{\beta}-\mathrm{j} G\right)\left(I+G^{2}\right)^{-\frac{1}{2}}\right) \leq 1
$$

Then for any real $\hat{\beta}>\beta$ there exist matrices $\hat{D} \in \mathcal{D}_{\mathcal{K}}$ and $\hat{G} \in \mathcal{G}_{\mathcal{K}}$ such that

$$
\bar{\sigma}\left(\left(\frac{\hat{D} M \hat{D}^{-1}}{\hat{\beta}}-\mathbf{j} \hat{G}\right)\left(I+\hat{G}^{2}\right)^{-\frac{1}{2}}\right)<1
$$


Proof: For convenience define $\hat{M} \doteq D M D^{-1}$, and we have

$$
\begin{aligned}
& \bar{\sigma}\left(\left(\frac{\hat{M}}{\beta}-\mathrm{j} G\right)\left(I+G^{2}\right)^{-\frac{1}{2}}\right) \leq 1 \\
& \left(\frac{\hat{M}^{*}}{\beta}+\mathrm{j} G\right)\left(\frac{\hat{M}}{\beta}-\mathrm{j} G\right) \leq I+G^{2} \\
& \longrightarrow \quad \hat{M}^{*} \hat{M}+\mathbf{j} \beta\left(G \hat{M}-\hat{M}^{*} G\right) \leq \beta^{2} I<\hat{\beta}^{2} I \\
& \longrightarrow \quad \frac{\hat{M}^{*} \hat{M}}{\hat{\beta}^{2}}+\mathrm{j} \frac{\beta}{\hat{\beta}^{2}}\left(G \hat{M}-\hat{M}^{*} G\right)<I
\end{aligned}
$$

Thus defining $\hat{D} \doteq D, \hat{G} \doteq \frac{\beta}{\hat{\beta}} G$ we have

$$
\begin{array}{cccc} 
& \frac{\hat{M}^{*} \hat{M}}{\hat{\beta}^{2}}+\frac{\mathbf{j}}{\hat{\beta}}\left(\hat{G} \hat{M}-\hat{M}^{*} \hat{G}\right) & < & I \\
\longrightarrow \quad & \left(\frac{\hat{M}^{*}}{\hat{\beta}}+\mathbf{j} \hat{G}\right)\left(\frac{\hat{M}}{\hat{\beta}}-\mathbf{j} \hat{G}\right) & < & I+\hat{G}^{2} \\
\longrightarrow \quad & \bar{\sigma}\left(\left(\frac{\hat{\bar{B}} \hat{D}^{-1}}{\hat{\beta}}-\mathbf{j} \hat{G}\right)\left(I+\hat{G}^{2}\right)^{-\frac{1}{2}}\right) & < & 1
\end{array}
$$

Theorem 7 Suppose we have matrices $M \in \mathbb{C}^{n \times n}, D \in \mathcal{D}_{\mathcal{K}}, G \in \mathcal{G}_{\mathcal{K}}$ and real scalars $\beta>0$ and $0<r \leq 1$ such that

$$
\bar{\sigma}\left(\left(\frac{D M D^{-1}}{\beta}-\mathrm{j} G\right)\left(I+G^{2}\right)^{-\frac{1}{2}}\right) \leq r
$$

Then there exists $\hat{D} \in \mathcal{D}_{\mathcal{K}}, \hat{G} \in \mathcal{G}_{\mathcal{K}}$ such that

$$
\bar{\sigma}\left(\left(\frac{\hat{D} M \hat{D}^{-1}}{r \beta}-\mathbf{j} \hat{G}\right)\left(I+\hat{G}^{2}\right)^{-\frac{1}{2}}\right) \leq 1
$$

Proof: Now define $\hat{M} \doteq \frac{D M D^{-1}}{\beta}$ and we have

$$
\begin{aligned}
& \bar{\sigma}\left((\hat{M}-\mathbf{j} G)\left(I+G^{2}\right)^{-\frac{1}{2}}\right) \leq r \\
& \longrightarrow \quad\left(\hat{M}^{*}+\mathrm{j} G\right)(\hat{M}-\mathrm{j} G) \quad \leq \quad r^{2}\left(I+G^{2}\right) \\
& \longrightarrow \hat{M}^{*} \hat{M}+\mathrm{j}\left(G \hat{M}-\hat{M}^{*} G\right) \leq r^{2} I+\left(r^{2}-1\right) G^{2} \leq r^{2} I \\
& \longrightarrow \quad \frac{\hat{M}^{*} \hat{M}}{r^{2}}+\frac{j}{r^{2}}\left(G \hat{M}-\hat{M}^{*} G\right) \leq I
\end{aligned}
$$

so that defining $\hat{D} \doteq D, \hat{G} \doteq \frac{G}{r}$ we have

$$
\begin{aligned}
& \frac{\hat{M}^{*} \hat{M}}{r^{2}}+\frac{\mathbf{j}}{r}\left(\hat{G} \hat{M}-\hat{M}^{*} \hat{G}\right) \leq I \\
& \left(\frac{\hat{M}^{*}}{r}+\mathbf{j} \hat{G}\right)\left(\frac{\hat{M}}{r}-\mathbf{j} \hat{G}\right) \leq I+\hat{G}^{2} \\
& \longrightarrow \quad \bar{\sigma}\left(\left(\frac{\hat{D} M \hat{D}^{-1}}{r \beta}-\mathrm{j} \hat{G}\right)\left(I+\hat{G}^{2}\right)^{-\frac{1}{2}}\right) \leq 1
\end{aligned}
$$

These two theorems tell us something about how the mixed $\mu$ upper bound scales. Note that both of these results are trivial in the complex case, where $G=0_{n}$. For the mixed case however the results are not so obvious, and in particular it is important to note that theorem 7 applies only if $r \leq 1$. From this theorem we see that whenever we have the appropriate scaling matrices and $r \leq 1$, then $r \beta$ is an upper bound for $\mu_{\mathcal{K}}(M)$. However when $r>1$ we cannot conclude that $r \beta$ is an upper bound in the mixed case, even though this conclusion is obviously true in the complex case. In fact it is easy to construct mixed examples with $r>1$ where $r \beta$ is strictly less than $\mu_{\mathcal{K}}(M)$.

We now propose the following " $D, G-K$ iteration" for the mixed $\mu$ synthesis problem: 


\section{Procedure 3 ( $D, G-K$ Iteration)}

1. Find initial estimates of the scaling matrices $D(\omega), G(\omega)$, and the real positive scalar $\beta_{*}$. One possibility for the scalings $D(\omega)$ is to choose them as the identity matrix at each frequency point. If $G(\omega)$ is chosen to be the zero matrix at each frequency point, then $\beta_{*}$ is arbitrary (so choose say $\beta_{*}=1$ ), otherwise it must satisfy

$$
\bar{\sigma}\left(\left(\frac{D(\omega) \mathbf{M}(\mathbf{P}, \mathbf{K})(\mathbf{j} \omega) D^{-1}(\omega)}{\beta_{*}}-\mathbf{j} G(\omega)\right)\left(I+G(\omega)^{2}\right)^{-\frac{1}{2}}\right) \leq 1
$$

for all $\omega$, for some stabilizing controller $\mathbf{K}$.

2. Fit State Space realizations $\mathbf{D}$ and $\mathbf{G}$ to the pointwise scaling matrices $D(\omega)$ and $\mathbf{j} G(\omega)$, so that $\mathbf{D}(\mathbf{j} \omega)$ approximates $D(\omega)$, and $\mathbf{G}(\mathbf{j} \omega)$ approximates $\mathbf{j} G(\omega)$. Now replace $\mathbf{D}$ and $\mathbf{G}$ with appropriate factors so that $\mathbf{D}, \mathbf{D}^{-1}, \mathbf{G}_{h}$, and $\mathbf{G G}_{h}$ are all stable, where $\mathbf{G}_{h}$ is a spectral factor satisfying $\left(I+\mathbf{G}^{\sim} \mathbf{G}\right)^{-1}=\mathbf{G}_{h} \mathbf{G}_{h}^{\sim}$. Augment $\mathbf{D}$ and $\mathbf{G}_{h}$ with identity matrices, and $\mathbf{G}$ with a zero matrix, of appropriate dimensions so that $\mathbf{D}, \mathbf{G}, \mathbf{G}_{h}$ are all compatible with $\mathbf{P}$. Form the State Space system $\mathbf{P}_{\mathbf{D G}}=\left(\mathbf{D P D} \mathbf{D}^{-1}-\beta_{*} \mathbf{G}\right) \mathbf{G}_{h}$.

3. Find the $\mathcal{H}_{\infty}$ optimal controller $\hat{\mathbf{K}}$ minimizing $\left\|\mathbf{M}\left(\mathbf{P}_{\mathbf{D G}}, \mathbf{K}\right)\right\|_{\infty}$ over all all stabilizing, proper, real rational controllers $\mathbf{K}$.

4. Compute $\beta_{*}$ as

$$
\beta_{*}=\sup _{\omega \in \mathbb{R}} \inf _{\tilde{D}(\omega) \in \mathcal{D}_{\mathcal{K}}, \tilde{G}(\omega) \in \mathcal{G}_{\mathcal{K}}} \inf _{\beta(\omega) \in \mathbb{R}, \beta(\omega)>0}\{\beta(\omega): \Gamma \leq 1\}
$$

where

$$
\Gamma=\bar{\sigma}\left(\left(\frac{\tilde{D}(\omega) \mathbf{M}(\mathbf{P}, \mathbf{K})(\mathbf{j} \omega) \tilde{D}^{-1}(\omega)}{\beta(\omega)}-\mathrm{j} \tilde{G}(\omega)\right)\left(I+\tilde{G}^{2}(\omega)\right)^{-\frac{1}{2}}\right)
$$

5. Find $\hat{D}(\omega), \hat{G}(\omega)$ solving the minimization problem

$$
\inf _{\hat{D}(\omega) \in \mathcal{D}_{\kappa}, \hat{G}(\omega) \in \mathcal{G}_{\kappa} .} \bar{\sigma}\left(\left(\frac{\hat{D}(\omega) \mathbf{M}(\mathbf{P}, \mathbf{K})(\mathbf{j} \omega) \hat{D}^{-1}(\omega)}{\beta_{*}}-\mathbf{j} \hat{G}(\omega)\right)\left(I+\hat{G}^{2}(\omega)\right)^{-\frac{1}{2}}\right)
$$

pointwise across frequency.

6. Compare the new scaling matrices $\hat{D}(\omega), \hat{G}(\omega)$ with the previous estimates $D(\omega), G(\omega)$. Stop if they are close, else replace $D(\omega), G(\omega)$ with $\hat{D}(\omega), \hat{G}(\omega)$ respectively and return to step 2 .

We will see that this iteration (assuming perfect State Space realizations of $D(\omega)$ and $G(\omega)$ ) is monotonically nonincreasing, so that we are guaranteed convergence to a local minimum of the problem. Having converged to such a point then the controller $\hat{\mathbf{K}}$ from step 3 is the resulting mixed $\mu$ synthesis controller, and it satisfies

$$
\sup _{\omega \in \mathbb{R}} \mu_{\mathcal{K}}(\mathbf{M}(\mathbf{P}, \hat{\mathbf{K}})(\mathbf{j} \omega)) \leq \beta_{*}
$$

Note that, as in the complex $\mu$ case, we use the $\mathcal{H}_{\infty}$ optimal control solution to synthesize the controller. By construction the scaled system P $_{\mathbf{D G}}$ fits into the standard $\mathcal{H}_{\infty}$ optimal control framework, as shown in figure 3 . 


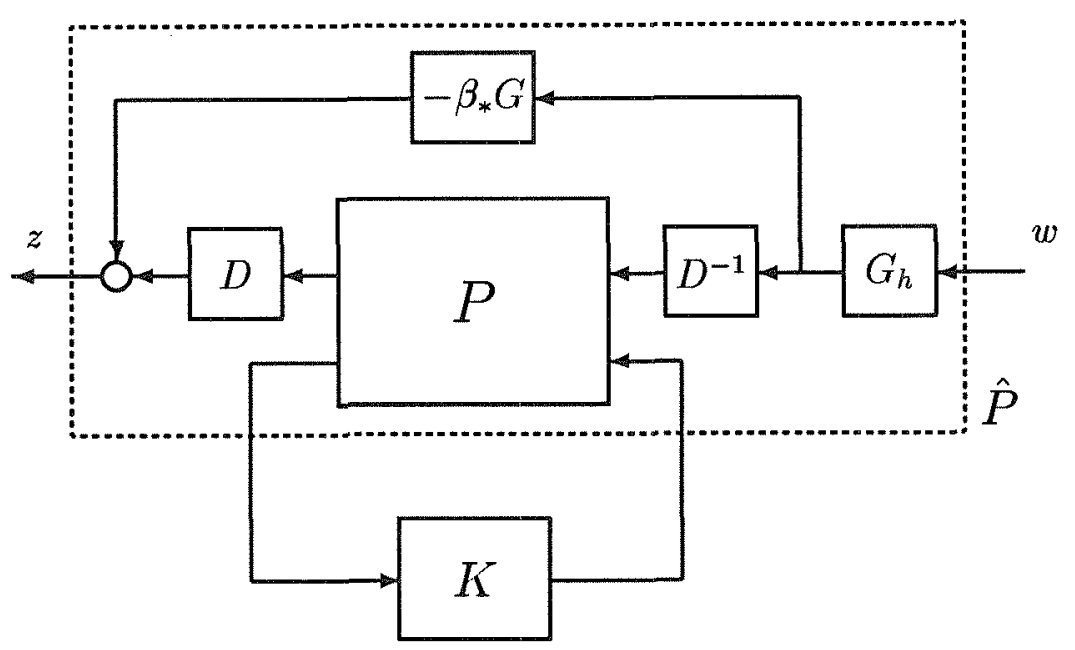

Figure 3: The " $K$ " iteration of the " $D, G-K$ iteration" procedure

The fact that this iteration is monotonically nonincreasing follows from theorems 6 and 7 in the following way. Suppose we have run through several cycles of the above procedure, and have arrived at step 3 , where we wish to design a new controller. Since $\beta_{*}$ was defined in (the previous) step 4 as the maximum across frequency of the upper bound we have that $\beta_{*} \geq \beta(\omega)$ for all $\omega$, with $\beta_{*}=\beta(\omega)$ only for those frequency points whose upper bound achieves the maximum across frequency. Then theorem 6 guarantees us that in (the previous) step 5 we could achieve

$$
\bar{\sigma}\left(\left(\frac{\hat{D}(\omega) \mathbf{M}(\mathbf{P}, \mathbf{K})(\mathbf{j} \omega) \hat{D}^{-1}(\omega)}{\beta_{*}}-\mathbf{j} \hat{G}(\omega)\right)\left(I+\hat{G}^{2}(\omega)\right)^{-\frac{1}{2}}\right)<1
$$

for all frequency points with $\beta_{*}>\beta(\omega)$ and

$$
\bar{\sigma}\left(\left(\frac{\hat{D}(\omega) \mathbf{M}(\mathbf{P}, \mathbf{K})(\mathbf{j} \omega) \hat{D}^{-1}(\omega)}{\beta_{*}}-\mathbf{j} \hat{G}(\omega)\right)\left(I+\hat{G}^{2}(\omega)\right)^{-\frac{1}{2}}\right)=1
$$

for points with $\beta_{*}=\beta(\omega)$. Now our previous controller from step $3, \hat{\mathbf{K}}$, stabilized the interconnection $\mathbf{M}(\mathbf{P}, \hat{\mathbf{K}})$, and hence, by our choice of factorizations for $\mathbf{D}$ and $\mathbf{G}_{h}$, $\hat{\mathbf{K}}$ stabilizes $\mathbf{M}\left(\mathbf{P}_{\mathbf{D G}}, \hat{\mathbf{K}}\right)$ as well. Note that the above formulae imply that $\hat{\mathbf{K}}$ achieved $\left\|\mathbf{M}\left(\mathbf{P}_{\mathbf{D G}}, \hat{\mathbf{K}}\right)\right\|_{\infty}=\beta_{*}$, so that we are guaranteed the existence of a stabilizing controller achieving at least this level for $\left\|\mathbf{M}\left(\mathbf{P}_{\mathbf{D G}}, \hat{\mathbf{K}}_{n e w}\right)\right\|_{\infty}$. Thus our new $\mathcal{H}_{\infty}$ optimal controller in step 3 will satisfy $\left\|\mathbf{M}\left(\mathbf{P}_{\mathbf{D G}}, \hat{\mathbf{K}}_{n e w}\right)\right\|_{\infty}=$ $r \beta_{*}$ with $r \leq 1$. But theorem 7 implies that $r \beta_{*}$ is now an upper bound for $\mu$ across frequency, and so the new computation of $\beta_{* n e w}$ in step 4 will yield $\beta_{* n e w} \leq r \beta_{*} \leq \beta_{*}$. Thus the iteration is monotonically nonincreasing, and furthermore if the new $\mathcal{H}_{\infty}$-optimal controller from step 3 achieves any improvement over the old one (i.e., $r<1$ ) then the peak value of the $\mu$ upper bound across frequency is strictly decreased.

Note also that the $\mathcal{H}_{\infty}$ norm of the interconnection $\mathbf{M}\left(\mathbf{P}_{\mathbf{D G}}, \hat{\mathbf{K}}\right)$ (formed by closing the new interconnection $\mathbf{P}_{\text {DG }}$ from step 2 with the previous controller $\hat{\mathbf{K}}$ ) is determined by those frequencies 
which have the maximum value for the mixed $\mu$ upper bound. This places the emphasis for the $\mathcal{H}_{\infty}$ norm minimization at the frequencies where mixed $\mu$ is largest (which is just what we want). Furthermore the way we have constructed the interconnection $\mathbf{P}_{\mathbf{D G}}$ guarantees that if the new controller $\hat{\mathbf{K}}_{n e w}$ reduces the $\mathcal{H}_{\infty}$ norm of $\mathbf{M}\left(\mathbf{P}_{\mathbf{D G}}, \hat{\mathbf{K}}_{n e w}\right)$, then the peak value of the $\mu$ upper bound across frequency is reduced.

Note that in step 4 we compute scalings $\tilde{D}(\omega), \tilde{G}(\omega)$ which minimize the scalar $\beta(\omega)$ at each frequency subject to a (scaled) singular value constraint, and this is exactly the mixed $\mu$ upper bound computation across frequency. A closely related problem is solved in step 5, where we compute scalings $\hat{D}(\omega), \hat{G}(\omega)$ to minimize a (scaled) singular value problem with $\beta_{*}$ fixed across frequency. If we consider complex problems then the separate computation of $\tilde{D}(\omega), \tilde{G}(\omega)$ in step 4 , and then $\hat{D}(\omega), \hat{G}(\omega)$ in step 5 is unnecessary, since it is easy to see that the two sets of optimal scalings may be taken to be the same. For mixed problems however the two sets of scalings in steps 4 and 5 can be quite different, and it is important to use the scalings $\hat{D}(\omega), \hat{G}(\omega)$ from step 5 for fitting (in step 2).

\subsubsection{Forming The New Interconnection}

In order to implement the " $D, G-K$ iteration" described in the preceding subsection we need to be able to carry out certain factorizations, required in step 2. These factorizations are used to ensure that if $\hat{\mathbf{K}}$ stabilizes $\mathbf{M}(\mathbf{P}, \hat{\mathbf{K}})$, then $\hat{\mathbf{K}}$ also stabilizes $\mathbf{M}\left(\left(\mathbf{D P D}^{-1}-\beta_{*} \mathbf{G}\right) \mathbf{G}_{h}, \hat{\mathbf{K}}\right)$, and vica versa (for the converse direction we need a slight technical assumption that we don't get right half-plane pole/zero cancellations when forming $\mathbf{D P D}^{-1} \mathbf{G}_{h}$ ). The fact that $\hat{\mathbf{K}}$ stabilizes both these interconnections was used when showing that the " $D, G-K$ iteration" is monotonically nonincreasing (and hence converges).

The problem of fitting pointwise frequency data $D(\omega), G(\omega)$ with State Space realizations $\mathbf{D}, \mathbf{G}$ will be considered in the next subsection. For now let us assume that we can obtain such fits. We will see in section 4.2.3 that we may assume that $\mathrm{D}$ is square and invertible with $\mathbf{D}, \mathbf{D}^{-1} \in \mathcal{R} \mathcal{L}_{\infty}$, but the only assumption we will make about $\mathbf{G}$ is that it is square.

Given State Space realizations for $\mathbf{D}$ and $\mathbf{G}$ we want to factor them as outlined in step 2 of the " $D, G-K$ iteration," described in procedure 3 , so that for the new $\mathbf{D}, \mathbf{G}, \mathbf{G}_{h}$ the interconnection $\mathbf{M}\left(\left(\mathbf{D P D} \mathbf{D}^{-1}-\boldsymbol{\beta}_{*} \mathbf{G}\right) \mathbf{G}_{h}, \hat{\mathbf{K}}\right)$ is stable, and we would like to do this without affecting the value of

$$
\bar{\sigma}\left(\mathbf{M}\left(\left(\mathbf{D}(\mathbf{j} \omega) \mathbf{P}(\mathbf{j} \omega) \mathbf{D}^{-1}(\mathbf{j} \omega)-\beta_{*} \mathbf{G}(\mathbf{j} \omega)\right) \mathbf{G}_{h}(\mathbf{j} \omega), \hat{\mathbf{K}}(\mathbf{j} \omega)\right)\right)
$$

for any frequency. This can be accomplished by using the machinery developed in section 3, applied to each block of $\mathbf{D}$ and $\mathbf{G}$.

First of all suppose we have fitted $D(\omega)$ with a transfer matrix D, but have not yet fitted $G(\omega)$. Apply theorem 4 to each block of $\mathbf{D}$ to factor out an all pass function so that the resulting block of $\mathbf{D}$ is stable minimum phase (hence $\mathbf{D}$ and $\mathbf{D}^{-1}$ are stable). Thus we have a new transfer matrix $\mathbf{D}_{s m p}$ which is stable minimum phase, and at each frequency point we have

$$
\mathrm{D}(\mathrm{j} \omega)=U(\omega) \mathbf{D}_{s m p}(\mathrm{j} \omega)
$$

where $U(\omega)$ is a unitary block diagonal matrix (with the same block structure as $\mathbf{D}$ ). Then denote $\mathbf{D}(\mathbf{j} \omega), U(\omega)$ and $\mathbf{D}_{s m p}(\mathrm{j} \omega)$ by $D_{w}, U_{w}$ and $D_{s w}$ respectively. Finally denote the pointwise frequency 
data $G(\omega)$ (to be fitted) by $G_{w}$, and we have the following equivalences:

$$
\left.\begin{array}{rl} 
& \bar{\sigma} \\
= & \bar{\sigma} \\
= & \bar{\sigma} \\
= & \bar{\sigma}
\end{array}\right\}\left(\begin{array}{l}
\left.\left.D_{w} \mathbf{M}(\mathbf{P}, \hat{\mathbf{K}})(\mathbf{j} \omega) D_{w}^{-1}-\mathbf{j} \beta_{*} G_{w}\right)\left(I+G_{w}^{2}\right)^{-\frac{1}{2}}\right) \\
\left.\left.U_{w} D_{s w} \mathbf{M}(\mathbf{P}, \hat{\mathbf{K}})(\mathbf{j} \omega) D_{s w}^{-1} U_{w}^{*}-\mathbf{j} \beta_{*} G_{w}\right)\left(I+G_{w}^{2}\right)^{-\frac{1}{2}}\right) \\
\left.\left.\left.D_{s w} \mathbf{M}, \hat{\mathbf{K}}\right)(\mathbf{j} \omega) D_{s w}^{-1}-\mathbf{j} \beta_{*} U_{w}^{*} G_{w} U_{w}\right) U_{w}^{*}\left(I+G_{w}^{2}\right)^{-\frac{1}{2}} U_{w}\right) \\
\left.\left.D_{s w} \mathbf{M}(\mathbf{P}, \hat{\mathbf{K}})(\mathbf{j} \omega) D_{s w}^{-1}-\mathbf{j} \beta_{*} \hat{G}_{w}\right)\left(I+\hat{G}_{w}^{2}\right)^{-\frac{1}{2}}\right)
\end{array}\right.
$$

where $\hat{G}_{w}=U_{w}^{*} G_{w} U_{w}$. Note that $\hat{G}_{w} \in \mathcal{G}_{\mathcal{K}}$, so now choose the transfer matrix $\mathbf{G}$ so that $\mathbf{G}(\mathbf{j} \omega)$ is a fit to $\mathbf{j} \hat{G}_{w}$ (rather than $\mathbf{j} G_{w}$ ). We may now apply theorem 5 to each non-zero block of $\mathrm{G}$ to construct $\mathbf{G}_{h}$, with $\mathbf{G}_{h}$ and $\mathbf{G G}_{h}$ stable, and $\mathbf{G}_{h}$ having the same block diagonal structure as $\mathbf{G}$. Furthermore at each frequency $\mathbf{G}_{h}$ satisfies

$$
\left(I+\mathbf{G}^{\sim}(\mathbf{j} \omega) \mathbf{G}(\mathbf{j} \omega)\right)^{-1}=\left(I+\hat{G}_{w}^{2}\right)^{-1}=\mathbf{G}_{h}(\mathbf{j} \omega) \mathbf{G}_{h}^{\sim}(\mathbf{j} \omega)
$$

Now for convenience denote $D_{s w} \mathbf{M}(\mathbf{P}, \hat{\mathbf{K}})(\mathrm{j} \omega) D_{s w}^{-1}$ by $M_{D_{w}}$, and $\mathbf{G}_{h}(\mathbf{j} \omega)$ by $G_{h w}$. Then note that we have

$$
\begin{aligned}
& \bar{\sigma}^{2}\left(\left(M_{D_{w}}-\mathbf{j} \beta_{*} \hat{G}_{w}\right)\left(I+\hat{G}_{w}^{2}\right)^{-\frac{1}{2}}\right) \\
= & \bar{\lambda}\left(\left(I+\hat{G}_{w}^{2}\right)^{-\frac{1}{2}}\left(M_{D_{w}}^{*}-\beta_{*} \mathbf{G}^{*}(\mathbf{j} \omega)\right)\left(M_{D_{w}}-\beta_{*} \mathbf{G}(\mathbf{j} \omega)\right)\left(I+\hat{G}_{w}^{2}\right)^{-\frac{1}{2}}\right) \\
= & \bar{\lambda}\left(\left(M_{D_{w}}^{*}-\beta_{*} \mathrm{G}^{*}(\mathbf{j} \omega)\right)\left(M_{D_{w}}-\beta_{*} \mathrm{G}(\mathbf{j} \omega)\right)\left(I+\hat{G}_{w}^{2}\right)^{-1}\right) \\
= & \bar{\lambda}\left(\left(M_{D_{w}}^{*}-\beta_{*} \mathrm{G}^{*}(\mathbf{j} \omega)\right)\left(M_{D_{w}}-\beta_{*} \mathrm{G}(\mathbf{j} \omega)\right) G_{h w} G_{h w}^{\sim}\right) \\
= & \bar{\lambda}\left(G_{h w}^{\sim}\left(M_{D_{w}}^{*}-\beta_{*} \mathbf{G}^{*}(\mathbf{j} \omega)\right)\left(M_{D_{w}}-\beta_{*} \mathbf{G}(\mathbf{j} \omega)\right) G_{h w}\right) \\
= & \bar{\sigma}^{2}\left(\left(M_{D_{w}}-\beta_{*} \mathrm{G}(\mathbf{j} \omega)\right) G_{h w}\right)
\end{aligned}
$$

so that this factorization does just what we require, and the appropriate State Space formulae for $\mathbf{D}, \mathbf{G}_{h}$ and $\mathbf{G G}_{h}$ may be obtained by applying the results from section 3 to each block of $\mathbf{D}$ and G.

Note that the final step of this process is to augment $\mathbf{D}$ and $\mathbf{G}_{h}$ with an identity matrix, and $\mathbf{G}$ with a zero matrix, of appropriate dimensions so that $\mathbf{D}, \mathbf{G}, \mathbf{G}_{h}$ are all compatible with $\mathbf{P}$, and then form the interconnection $\mathbf{P}_{\mathbf{D G}}=\left(\mathbf{D P D} \mathbf{D}^{-1}-\beta_{*} \mathbf{G}\right) \mathbf{G}_{h}$. Note that when forming $\mathbf{G G}_{h}$ we were able to employ state cancellations to obtain a stable realization with the same number of states as $\mathbf{G}_{h}$. It turns out that we may once again exploit the special structure of $\mathbf{G}$ and $\mathbf{G}_{h}$ to obtain further state cancellations when forming the interconnection $\mathbf{P}_{\mathbf{D G}}$. This state reduction comes about by cancelling uncontrollable and/or unobservable modes in the interconnection, and is highly desirable from a numerical viewpoint since the $\mathcal{H}_{\infty}$ optimal controller computed in step 3 of procedure 3 will have the same number of states as our realization for $\mathbf{P}_{\mathbf{D G}}$.

Note from theorem 5 that the State Space formulae for the blocks of $\mathbf{G}_{h}$ and $\mathbf{G G}_{h}$ share the same "A" and "B" matrices. In other words we have realizations for each block of $\mathbf{G}_{h}$ and $\mathbf{G G}_{h}$ which take the form

$$
\left(\mathbf{G}_{h}\right)_{i}=\left[\begin{array}{c|c}
A_{i} & B_{i} \\
\hline C_{i} & D_{i}
\end{array}\right] \quad\left(\mathbf{G G}_{h}\right)_{i}=\left[\begin{array}{c|c}
A_{i} & B_{i} \\
\hline \hat{C}_{i} & \hat{D}_{i}
\end{array}\right]
$$

Now that it is easy to show that since each block of $\mathbf{G}_{h}$ and $\mathbf{G G}_{h}$ satisfies these relationships, we may choose the block diagonal system realizations of $\mathbf{G}_{h}$ and $\mathbf{G G}_{h}$ to satisfy them as well. Furthermore 
we may scale $\mathrm{GG}_{h}$ by a real scalar (namely $-\beta_{*}$ ) and still preserve these relationships, since one may easily check that for any $\beta \in \mathbb{R}$

$$
\beta\left[\begin{array}{c|c}
A & B \\
\hline C & D
\end{array}\right]=\left[\begin{array}{c|c}
A & B \\
\hline \beta C & \beta D
\end{array}\right]
$$

Thus we may choose our realizations for $\mathbf{G}_{h}$ and $-\beta_{*} \mathbf{G G}_{h}$ to be of the form

$$
\mathbf{G}_{h}=\left[\begin{array}{c|c}
A_{G} & B_{G} \\
\hline C_{H} & D_{H}
\end{array}\right] \quad-\beta_{*} \mathbf{G G}_{h}=\left[\begin{array}{c|c}
A_{G} & B_{G} \\
\hline C_{G} & D_{G}
\end{array}\right]
$$

Now suppose that our State Space realization for $\mathbf{D P D} \mathbf{D}^{-1}$ is

$$
\mathrm{DPD}^{-1}=\left[\begin{array}{l|l}
A_{D} & B_{D} \\
\hline C_{D} & D_{D}
\end{array}\right]
$$

Then the interconnection $\mathbf{P}_{\mathbf{D G}}$ is given by

$$
\begin{aligned}
\mathbf{P}_{\mathbf{D G}} & = \\
& = \\
& =\left[\begin{array}{c|c|c|c}
A_{D} & B_{D} \\
\hline C_{D} & D_{D}
\end{array}\right]\left[\begin{array}{c|c}
A_{G} & B_{G} \\
\hline C_{H} & D_{H}
\end{array}\right]+\left[\begin{array}{ll|l}
A_{G} & B_{G} \\
\hline C_{G} & D_{G}
\end{array}\right] \\
& =\left[\begin{array}{ccc|c}
A_{D} & B_{D} C_{H} & 0 & B_{D} D_{H} \\
0 & A_{G} & 0 & B_{G} \\
0 & 0 & A_{G} & B_{G} \\
\hline C_{D} & D_{D} C_{H} & C_{G} & D_{D} D_{H}+D_{G}
\end{array}\right] \\
& =\left[\begin{array}{ccc|c}
A_{D} & B_{D} C_{H} & 0 & B_{D} D_{H} \\
0 & A_{G} & 0 & B_{G} \\
0 & 0 & A_{G} & 0 \\
\hline C_{D} & D_{D} C_{H}+C_{G} & C_{G} & D_{D} D_{H}+D_{G}
\end{array}\right] \\
& =\left[\begin{array}{cc|c}
A_{D} & B_{D} C_{H} & B_{D} D_{H} \\
0 & A_{G} & B_{G} \\
\hline C_{D} & D_{D} C_{H}+C_{G} & D_{D} D_{H}+D_{G}
\end{array}\right]
\end{aligned}
$$

where we have made use of the similarity transformation $T=\left(\begin{array}{ccc}I & 0 & 0 \\ 0 & I & 0 \\ 0 & I & I\end{array}\right)$. Note that in the final formula for $\mathbf{P}_{\mathbf{D G}}$ we only have the states from $\mathbf{D P D} \mathbf{D}^{-1}$ and $\mathbf{G}_{h}$ appearing once each.

\subsubsection{Fitting $D$ and $G$}

Finally we consider the problem of fitting State Space system realizations to frequency response data. Of course we will not attempt to exactly interpolate the data at each frequency point since that would require, in general, too many states. Rather we will attempt to obtain realizations that approximate the data over the frequency range of interest. Note that since we are concerned with the $\mathcal{H}_{\infty}$ norm of the resulting interconnection, we still require acceptable behavior from the State Space fits even outside the frequency range we are especially interested in. In particular we require that the fits behave reasonably as the frequency tends to zero or infinity. 
First of all consider the problem of fitting $D(\omega)$. This problem is encountered when implementing a " $D-K$ iteration" for complex $\mu$ synthesis, and hence has already received a good deal of attention. Several methods have been developed to tackle this problem, and although there is certainly room for improvement, they often work quite well.

In particular the $\mu$-Tools toolbox [BDG ${ }^{+91}$ contains two algorithms for fitting scalar magnitude data with a SISO system. One algorithm, fitmag, uses the complex-cepstrum (see [OS75]) to generate an appropriate phase, and then uses the algorithm invfreqs from the signal processing toolbox [LS88], which uses an equation error method to identify a system model from the frequency response data. Another $\mu$-Tools algorithm, fitmaglp, uses a linear programming technique to find a SISO system, which attempts to fit the magnitude data. In addition to these two algorithms for fitting magnitude data, there is the algorithm fitsys, which attempts to fit given scalar frequency response data in both magnitude and phase (with a SISO system). This algorithm is based heavily on the algorithm invfreqs (see [LS88] for more details).

Now one may easily build a routine to fit $D(\omega)$ by using the above algorithms to fit the (scalar) elements of $D(\omega)$ with SISO systems. The diagonal elements can be fitted (in magnitude only) with fitmag or fitmaglp, and the non-zero off diagonal elements can be fitted with fitsys. Putting these systems together appropriately we can form $\mathbf{D} \in \mathcal{R} \mathcal{M}$ fitting $D(\omega)$. This is the procedure we will use here, and we will not go into any further details, since it is the same procedure one typically uses in the complex $\mu$ synthesis case. Note that since $\mathbf{D}$ is a State Space system, $D(j \omega)$ tends to a real matrix as $\omega$ goes to zero or infinity, and of course we have that $\mathbf{M}(\mathbf{P}, \hat{\mathbf{K}})(\mathrm{j} \omega)$ also tends to a real matrix (for the same reason). The following theorem is easily shown from results in [You93].

Theorem 8 Given a matrix $M \in \mathbb{R}^{n \times n}$ and a compatible block structure $\mathcal{K}$ then we have that

$$
\begin{aligned}
& \inf _{D \in \mathcal{D}_{\mathcal{K}}, G \in \mathcal{G}_{\mathcal{K}}} \inf _{\beta \in \mathbb{R}, \beta>0}\left\{\beta: \bar{\sigma}\left(\left(\frac{D M D^{-1}}{\beta}-\mathrm{j} G\right)\left(I+G^{2}\right)^{-\frac{1}{2}}\right) \leq 1\right\} \\
= & \inf _{D \in \mathcal{D}_{\mathcal{K}}^{\mathrm{B}}, G \in \mathcal{G}_{\mathcal{K}}^{\mathbb{M}}} \inf _{\beta \in \mathbb{R}, \beta>0}\left\{\beta: \bar{\sigma}\left(\left(\frac{D M D^{-1}}{\beta}-\mathrm{j} G\right)\left(I+G^{2}\right)^{-\frac{1}{2}}\right) \leq 1\right\}
\end{aligned}
$$

where $\mathcal{D}_{\mathcal{K}}^{\mathbb{R}}, \mathcal{G}_{\mathcal{K}}^{\mathbb{R}}$ are defined as

$$
\begin{aligned}
\mathcal{D}_{\mathcal{K}}^{\mathbb{R}} & =\left\{D \in \mathcal{D}_{\mathcal{K}}: D \in \mathbb{R}^{n \times n}\right\} \\
\mathcal{G}_{\mathcal{K}}^{\mathbb{R}} & =\left\{G \in \mathcal{G}_{\mathcal{K}}: \mathrm{j} G \in \mathbb{R}^{n \times n}\right\}
\end{aligned}
$$

Proof: Follows immediately from the machinery of theorems 5.11 and 6.1 of [You93].

It follows from this result that the optimal scaling $D(\omega)$, at zero or infinite frequency, may be taken to be real as well. Thus the low/high frequency limits of $D(\omega)$ are amenable to fitting with a State Space system $\mathbf{D}(\mathrm{j} \omega)$. Note also that all blocks of $\mathrm{D}$ are square, and furthermore each block of $\mathbf{D}(\mathbf{j} \omega)$ may be taken to be Hermitian positive definite. Thus we do not need to allow sign changes in the (eigenvalues of the) blocks of $D(j \omega)$ and hence we do not need poles or zeros on the imaginary axis. Thus we may restrict our fitting routines to return $\mathbf{D} \in \mathcal{R} \mathcal{M}$ invertible, with $\mathbf{D}, \mathbf{D}^{-1} \in \mathcal{R} \mathcal{L}_{\infty}$ (which is typically done in the complex $\mu$ synthesis case, for the same reasons) as we required in section 4.2.2.

Now we turn our attention to the problem of fitting $\mathbf{j} G(\omega)$. Once again we will tackle this problem by fitting the (scalar) elements of $\mathrm{j} G(\omega)$ with SISO systems, and using these to build 
up G. For the non-zero off diagonal elements we can once again fit them using fitsys. Note that at zero or infinite frequency theorem 8 implies that the optimal $G(\omega)$ may be taken to be pure imaginary, so that $\mathrm{j} G(\omega)$ may be taken to be pure real, and hence amenable to fitting with a State Space system $\mathbf{G}(\mathrm{j} \omega)$. For the diagonal elements of $\mathrm{j} G(\omega)$ we have additional constraints, which are explored in more detail below.

Note that we need to fit $\mathbf{j} G(\omega)$ in both magnitude and phase. In particular we require that the phase of the diagonal elements of $\mathbf{G}$ is purely imaginary for all frequency. In order to see what constraints this places on $\mathbf{G}$ consider first the conditions under which a scalar transfer function $\mathbf{g}(s)$ is purely real for all frequency.

Lemma 6 Consider a scalar transfer function $\mathbf{g} \in \mathcal{R M}$. Then the following statements are equivalent

1. $\mathbf{g}(\mathbf{j} \omega)$ is purely real for all $\omega \in \mathbb{R}$.

2. $\mathbf{g}^{\sim}(s) \doteq \mathbf{g}(-s)=\mathbf{g}(s)$.

3. $\mathbf{g}(s)$ has pole and zero symmetry about the imaginary axis, with the total number of poles and zeros at the origin being even.

4. $\mathbf{g}(s)=\frac{z\left(s^{2}\right)}{p\left(s^{2}\right)}$ where $z\left(s^{2}\right)$ and $p\left(s^{2}\right)$ are polynomials in $s^{2}$.

Proof: We will prove that $1 \longrightarrow 2 \longrightarrow 3 \longrightarrow 4 \longrightarrow 1$. Suppose first $\mathbf{g}(\mathbf{j} \omega)$ is purely real for all $\omega \in \mathbb{R}$. Then

$$
\mathbf{g}(\mathbf{j} \omega)=\mathbf{g}^{*}(\mathbf{j} \omega)=\mathbf{g}(-\mathbf{j} \omega)
$$

and hence $\mathbf{g}(s)-\mathbf{g}(-s)$ is zero for all $s=\mathbf{j} \omega$. But since $(\mathbf{g}(s)-\mathbf{g}(-s)) \in \mathcal{R M}$ it has finitely many zeros and hence we must have $\mathbf{g}(s)-\mathbf{g}(-s)=0$, or in other words $\mathbf{g}^{\sim}(s) \doteq \mathbf{g}(-s)=\mathbf{g}(s)$. Now $\mathbf{g}(s)=\mathbf{g}(-s)$ gives that whenever $s_{0}$ is a zero (pole) of $\mathbf{g}(s)$, then $-s_{0}$ is a zero (pole) of $\mathbf{g}(s)$. Together with the fact that for $\mathbf{g} \in \mathcal{R} \mathcal{M}$ the poles and zeros come in conjugate symmetric pairs, this implies that we have pole and zero symmetry about the imaginary axis. Since this implies that all the poles and zeros not at the origin must come in pairs (or fours), it is easy to check that we must also have the total number of poles and zeros at the origin being even. By collecting terms in pairs it is easy to check that, if we have pole and zero symmetry about the imaginary axis, and the total number of poles and zeros at the origin is even, then we may write $\mathbf{g}(s)=\frac{z\left(s^{2}\right)}{p\left(s^{2}\right)}$. Finally it is clear that if $\mathbf{g}(s)=\frac{z\left(s^{2}\right)}{p\left(s^{2}\right)}$ then $\mathbf{g}(\mathbf{j} \omega)$ is purely real for all $\omega \in \mathbb{R}$.

A simple extension of these arguments gives us the conditions under which $\mathbf{g}(s)$ is purely imaginary for all frequency.

Lemma 7 Consider a scalar transfer function $\mathrm{g} \in \mathcal{R} \mathcal{M}$. Then the following statements are equivalent

1. $\mathbf{g}(\mathbf{j} \omega)$ is purely imaginary for all $\omega \in \mathbb{R}$.

2. $\mathrm{g}^{\sim}(s) \doteq \mathrm{g}(-s)=-\mathrm{g}(s)$.

3. $\mathbf{g}(s)$ has pole and zero symmetry about the imaginary axis, with the total number of poles and zeros at the origin being odd. 
4. $\mathbf{g}(s)=s \frac{z\left(s^{2}\right)}{p\left(s^{2}\right)}$ where $z\left(s^{2}\right)$ and $p\left(s^{2}\right)$ are polynomials in $s^{2}$.

Proof: Again we prove that $1 \longrightarrow 2 \longrightarrow 3 \longrightarrow 4 \longrightarrow 1$. First suppose that $\mathbf{g}(\mathbf{j} \omega)$ is purely imaginary for all $\omega \in \mathbb{R}$. Then

$$
\mathrm{g}(\mathrm{j} \omega)=-\mathrm{g}^{*}(\mathrm{j} \omega)=-\mathrm{g}(-\mathbf{j} \omega)
$$

and hence $\mathbf{g}(s)+\mathbf{g}(-s)$ is zero for all $s=\mathbf{j} \omega$. But since $(\mathrm{g}(s)+\mathbf{g}(-s)) \in \mathcal{R M}$ it has finitely many zeros and hence we must have $\mathbf{g}(s)+\mathbf{g}(-s)=0$, or in other words $\mathbf{g}^{\sim}(s) \doteq \mathbf{g}(-s)=-\mathbf{g}(s)$. Now $\mathbf{g}(s)=-\mathbf{g}(-s)$ gives that whenever $s_{0}$ is a zero (pole) of $\mathbf{g}(s)$, then $-s_{0}$ is a zero (pole) of $\mathbf{g}(s)$. Together with the fact that for $g \in \mathcal{R} \mathcal{M}$ the poles and zeros come in conjugate symmetric pairs, this implies that we have pole and zero symmetry about the imaginary axis. Since this implies that all the poles and zeros not at the origin must come in pairs (or fours), it is easy to check that we must also have the total number of poles and zeros at the origin being odd. By collecting terms in pairs it is easy to check that, if we have pole and zero symmetry about the imaginary axis, and the total number of poles and zeros at the origin is odd, then we may write $g(s)=s \frac{z\left(s^{2}\right)}{p\left(s^{2}\right)}$. Finally it is clear that if $\mathbf{g}(s)=s \frac{z\left(s^{2}\right)}{p\left(s^{2}\right)}$ then $\mathbf{g}(\mathbf{j} \omega)$ is purely imaginary for all $\omega \in \mathbb{R}$.

Thus we see that a purely imaginary transfer function is obtained by adding a zero at the origin to a purely real transfer function. Our algorithm to fit purely imaginary SISO transfer functions, fitmagreal, which is used to fit the diagonal elements of $\mathrm{j} G(\omega)$, exploits this fact and is described briefly below.

Suppose that $g(\omega)$ is a diagonal element of $G(\omega)$. Then we first fit $h(\omega) \doteq \frac{g(\omega)}{\omega}$ with a purely real SISO transfer function, $\hat{\mathbf{g}}$. In order to do this we parametrize our allowable transfer functions for the fit as

$$
\hat{\mathrm{g}}(s)=\frac{z\left(s^{2}\right)}{p\left(s^{2}\right)}
$$

where the degree of $p\left(s^{2}\right)$ is chosen by the user, and the degree of $z\left(s^{2}\right)$ is chosen so that $\hat{\mathbf{g}}(s)$ is strictly proper. Thus we are left with the problem of finding the coefficients of $z$ and $p$ so that $\hat{\mathbf{g}}(\mathbf{j} \omega)=\frac{z\left(-w^{2}\right)}{p\left(-w^{2}\right)}$ best approximates $h(\omega)$. Note that all the quantities concerned in this problem are real numbers. We tackle this problem using an algorithm realfit, which is a modification of the algorithm fitmaglp, and is based on a linear programming approach. Note that $h(\omega)$ is not constrained in sign, so that we wish to allow sign changes in $\hat{\mathbf{g}}(\mathbf{j} \omega)$. Hence we do not place any restrictions on the pole/zero locations of $\hat{\mathbf{g}}(s)$, and in particular it may have poles and/or zeros on the imaginary axis.

Having obtained $\hat{\mathbf{g}}(s)$ as a strictly proper transfer function, say

$$
\hat{\mathrm{g}}=\left[\begin{array}{c|c}
A & B \\
\hline C & 0
\end{array}\right]
$$

then we form $\mathbf{g}$ as

$$
\mathbf{g}(s) \doteq s \hat{\mathrm{g}}(s) \quad \longrightarrow \quad \mathrm{g}=\left[\begin{array}{c|c}
A & B \\
\hline C A & C B
\end{array}\right]
$$

Thus we obtain $\mathbf{g}$ as a purely imaginary transfer function, with $\mathbf{g}(\mathbf{j} \omega)$ approximating $\mathbf{j} g(\omega)$. Note from this construction that the transfer matrix $\mathbf{G}$, built from these transfer functions, may contain poles and zeros located on the imaginary axis. For this reason the factorization of $\mathbf{G}$ used in section 4.2.2 did not make any assumptions about the pole/zero locations of $\mathbf{G}$. 
Note that lemma 7 implies that any non-zero purely imaginary (proper) transfer function $\mathbf{g} \epsilon$ $\mathcal{R M}$ is in fact strictly proper, and indeed our construction for $\mathbf{g}$ will yield a strictly proper transfer function. Thus $\mathbf{g}(\mathbf{j} \omega)$ tends to zero as $\omega$ tends to infinity. Furthermore, assuming $\hat{\mathbf{g}}(s)$ has no poles at the origin, then $g(s)$ has a zero at the origin, so that $g(j \omega)$ also tends to zero as $\omega$ tends to zero. Now by earlier arguments we know that theorem 8 implies that the optimal $G(\omega)$ may be taken to be purely imaginary at zero or infinite frequency. But $G(\omega)$ is Hermitian and so the diagonal elements are purely real, and hence the diagonal elements of the optimal $G(\omega)$ may be taken to be zero at zero or infinite frequency. Thus the high and low frequency limits of $\mathbf{g}(\mathbf{j} \omega)$ are compatible with the data it is attempting to fit (i. e., both $\mathbf{j} g(\omega)$ and $\mathrm{g}(\mathrm{j} \omega)$ tend to zero at high/low frequency).

Finally we note that since the routine fitsys can be used to fit a SISO transfer function in both magnitude and phase, we could apply this algorithm directly to the problem of fitting the diagonal elements of $G(\omega)$, rather than the approach taken above. Note however that the above approach enforces the phase constraints for all frequency, and enforces the correct behavior at the high and low frequency limits. We would not obtain these guarantees by using fitsys. Furthermore by simply applying fitsys we would not be exploiting all the information that we have about the structure of $\mathbf{g}$ (as is done above), and in fact we found that the fitsys routine performed poorly for many problems when fitmagreal was able to obtain quite good fits.

\subsubsection{Some Practical Considerations}

These procedures have been implemented in software as Matlab functions (m-files) "rmufit" and "rmuflp." Together with the mixed $\mu$ analysis software, "rmu" (see [YND92]), they allow the user to implement the " $D, G-K$ iteration" described in the preceding subsections, and hence to design mixed $\mu$ synthesis controllers.

It should be noted that for complex $\mu$ problems then the " $D, G-K$ iteration" reduces exactly to the familiar " $D-K$ iteration" for complex $\mu$ synthesis. Thus many of the techniques required to obtain a practical design from a complex $\mu$ synthesis procedure carry over into the mixed case as well. There are many issues to be considered, and we will not go into any of the details here, except to make the following comments. The procedures developed in this paper did not make any restrictions on the block structure, $\mathcal{K}$, of the uncertainties in the underlying robustness problem. In particular repeated scalar blocks (real or complex) were allowed. It should be noted however that for real or complex $\mu$ problems the presence of repeated scalar uncertainty blocks gives rise to full blocks in the scaling matrices $(D(\omega), G(\omega))$. Since the present approach fits element by element, then this may well require a lot of of states, and this approach may become impractical.

Fortunately for many problems we do not need repeated scalar uncertainty blocks, and so we only need diagonal scaling matrices. In fact the currently available software in the $\mu$-Tools toolbox (for complex $\mu$ synthesis) only handles this case. For this reason most of the effort in our approach has been to develop a method which will work well for that case (hence the emphasis on developing good routines for fitting the diagonal elements of $D(\omega)$ and $\mathrm{j} G(\omega)$ ). A practical solution to the more general case is a subject of ongoing research.

\section{Examples and Applications}

Finally we conclude the paper with a look at two simple design problems involving real parametric uncertainty. In both cases it will be seen that a simple application of complex $\mu$ synthesis techniques 
is not adequate, but the new mixed $\mu$ synthesis procedure performs well.

\section{Optimal Gain Margin Problem}

The problem of designing a controller to maximize the gain margin of a SISO plant can be solved analytically (see section 11.3 of [DFT92]). This question can (almost) be restated as a special case of a mixed $\mu$ synthesis problem, so that it provides us with a simple benchmark test for the " $D, G-K$ iteration" procedure.

Consider the feedback interconnection in figure 4 . The perturbed plant $\tilde{P}$ is given by

$$
\tilde{P}(s)=\left(\frac{s-1.2}{1-1.2 s}\right)\left(1+\delta^{r}\right)
$$

where $\delta^{r} \in \mathbb{R}$ is a real scalar uncertainty. This is a loopshaping set-up, and fits into the standard mixed $\mu$ synthesis framework of figure 1 . If we choose the performance weights for sensitivity and complementary sensitivity as

$$
W_{S_{1}}=W_{S_{2}}=W_{T_{1}}=W_{T_{2}}=0.01
$$

then the performance requirements for this problem are negligible. Thus we find that this robust performance problem is effectively a robust stability problem, and is in fact (approximately) the optimal gain margin problem.

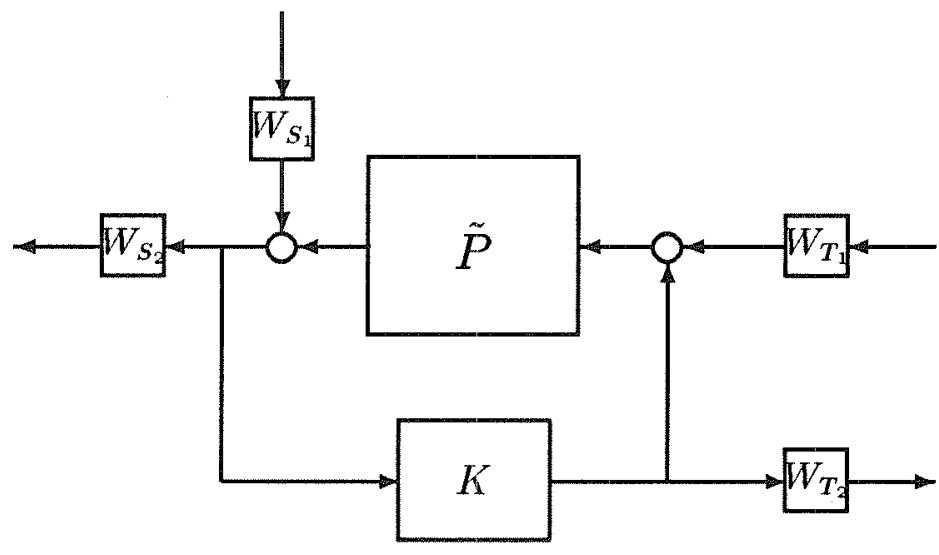

Figure 4: Interconnection for mixed $\mu$ synthesis examples

Note that the nominal plant is non-minimum phase and unstable. It can be stabilized simply with unity negative feedback, and the Nyquist plot is shown in figure 5 (for $\delta^{r}=0$ ). In addition the mixed and complex $\mu$ plots across frequency for the closed loop system are also shown. Note that in this example the mixed $\mu$ upper and lower bounds were tight, so that only the upper bounds are shown. For a plant of the form

$$
P=\frac{s-\alpha}{1-\alpha s}
$$


with $\alpha>1$, then the optimal gain margin controller is given by [Doy92]

$$
K=-\frac{1+\beta s}{s+\beta}
$$

where $\beta \downarrow \frac{1}{\alpha}$. The results for the optimal gain margin controller are shown in figure 6 , and it can be seen that this controller flattens out the Nyquist curve so as to substantially improve the gain margin. As a result the peak value across frequency of mixed $\mu$ is substantially improved (from 6.0 to 3.35 ), at the expense of complex $\mu$.
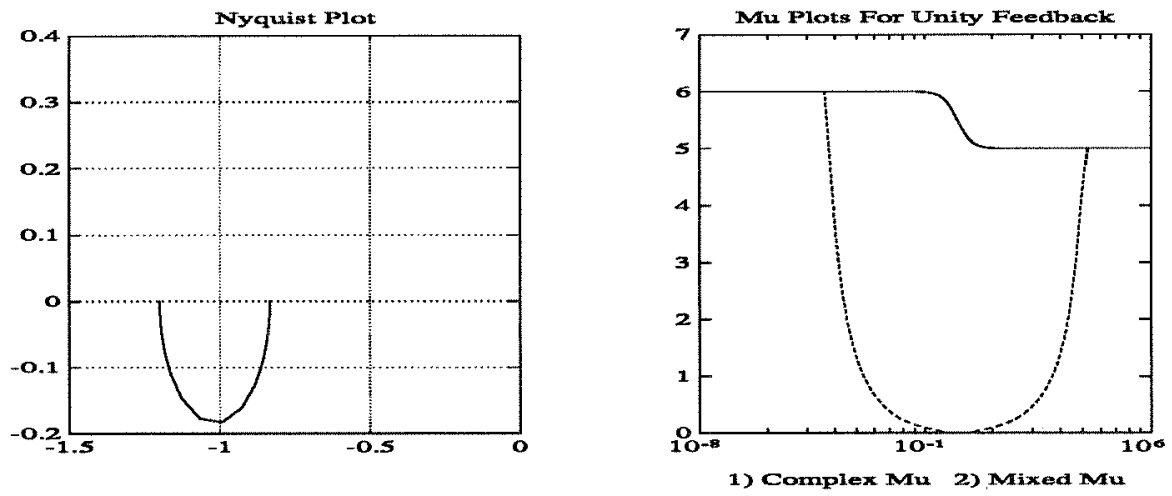

Figure 5: Nyquist and $\mu$ plots for unity (negative) feedback controller (gain margin problem)
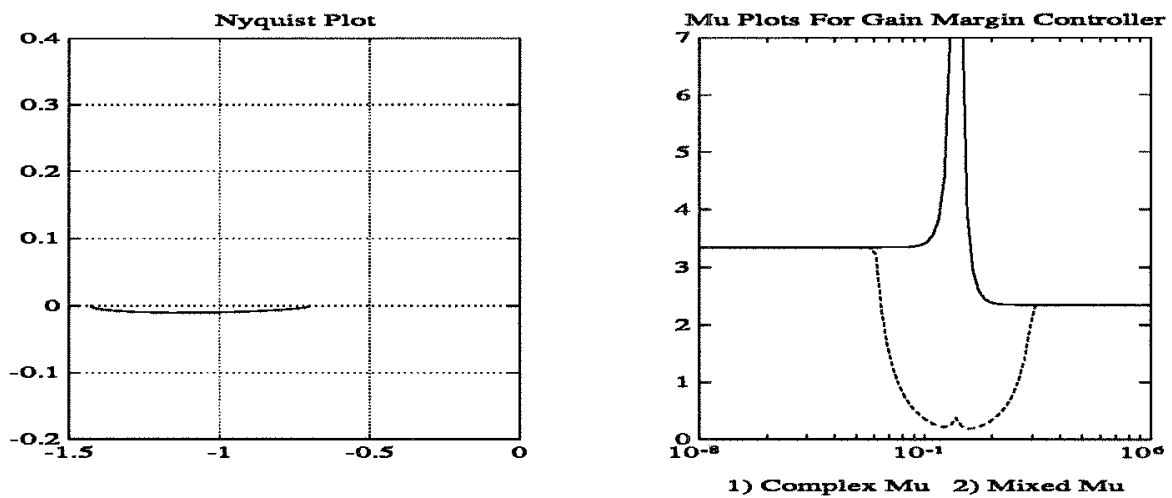

Figure 6: Nyquist and $\mu$ plots for optimal gain margin controller (gain margin problem)

The standard " $D$ - $K$ iteration" procedure for complex $\mu$ synthesis was applied to this problem, and the results are shown in figure 7 . Note that this controller gives a slight improvement over unity feedback for both mixed and complex $\mu$ (from 6.0 to 5.57). However the Nyquist plot is not greatly modified (other than the roll off at high frequency), so that the gain margin is not greatly improved. Applying the mixed $\mu$ synthesis procedure to this problem results in the plots in figure 8. Note that this controller achieves about the same level for mixed $\mu$ as the optimal (gain margin) controller. Once again this is achieved by flattening out the Nyquist curve so as to improve the gain margin at the expense of complex $\mu$. For this simple example we see that the mixed $\mu$ synthesis 
procedure does exactly the required thing, and shapes the frequency response so as to minimize the peak value across frequency of mixed $\mu$.
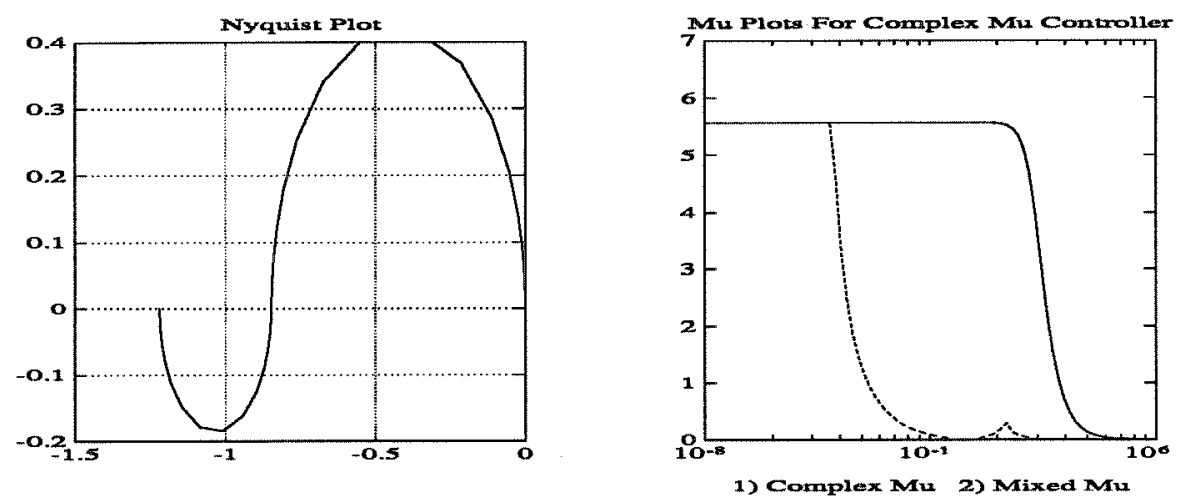

Figure 7: Nyquist and $\mu$ plots for complex $\mu$ synthesis controller (gain margin problem)
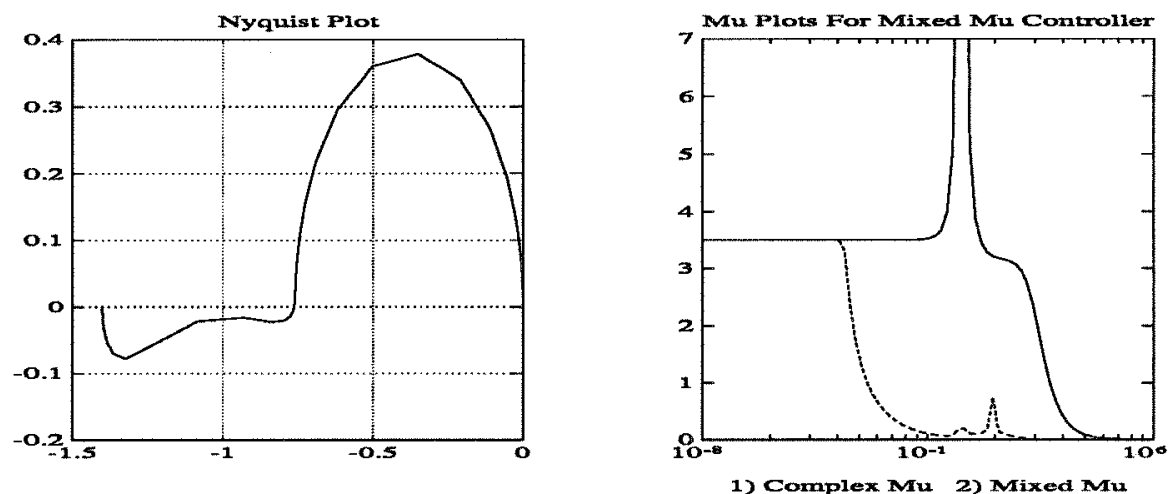

Figure 8: Nyquist and $\mu$ plots for mixed $\mu$ synthesis controller (gain margin problem)

\section{A Modal Example}

The previous example involved only one uncertain real parameter, so that it was possible to exploit our knowledge of the problem, and find a good controller design by another method for the purposes of comparison. We found that the mixed $\mu$ synthesis routine was able to find a comparable design. Of course the main point in developing the mixed $\mu$ synthesis machinery was to have a systematic design procedure, capable of dealing with more complicated problems, where alternative approaches are no longer available. We conclude the paper with a synthesis example for a problem with two uncertain real parameters.

Consider once again the interconnection in figure 4. As in the gain margin problem we make this essentially a robust stability problem by choosing the weights as

$$
W_{S_{1}}=W_{S_{2}}=W_{T_{1}}=W_{T_{2}}=0.01
$$


so that the performance requirements are negligible. Now consider an uncertain plant of the form

$$
\tilde{P}(s)=\frac{s^{2}-2 \zeta \omega s+\omega^{2}\left(1+\delta_{1}^{r}\right)}{s^{2}+2 \zeta \omega s+\omega^{2}\left(1+\delta_{2}^{r}\right)}
$$

where $\delta_{1}^{r}, \delta_{2}^{r} \in \mathbb{R}$ are uncertain real parameters. This is a second-order transfer function intended to represent a lightly damped mode in a flexible structure. This can be put in standard $\mu$ synthesis form by rewriting as $\tilde{P}$ as in figure 9 where $\Delta=\operatorname{diag}\left(\delta_{1}^{r}, \delta_{2}^{r}\right)$ and

$$
G=\left[\begin{array}{cc|ccc}
0 & 1 & -\frac{1}{\omega^{2}} & -\frac{1}{\omega^{2}} & 0 \\
-\omega^{2} & -2 \zeta \omega & 0 & 0 & -4 \zeta \omega \\
\hline 0 & 0 & 0 & 0 & \omega^{2} \\
0 & -\omega^{2} & 0 & 0 & -\omega^{2} \\
0 & 1 & 0 & 0 & 1
\end{array}\right]
$$

For this example we choose $\omega=1$ and $\zeta=0.2$, so that the mode is lightly damped, with a pair of

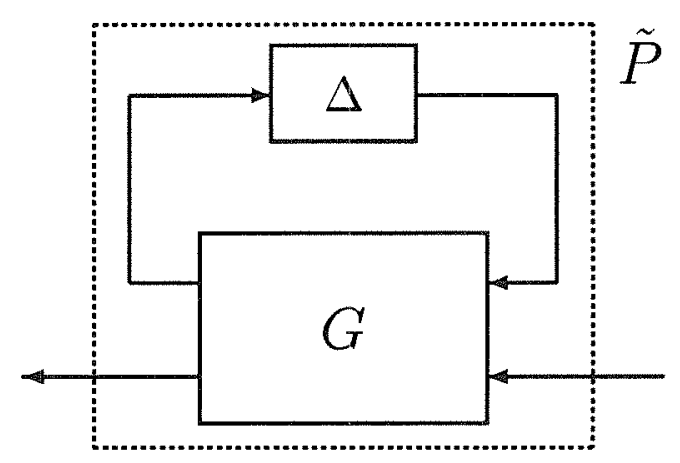

Figure 9: Modal example uncertain plant

complex conjugate poles at $-0.2 \pm 0.9798 \mathbf{j}$ and a pair of complex conjugate zeros at $0.2 \pm 0.9798 \mathbf{j}$ $\left(\right.$ for $\delta_{1}^{r}=\delta_{2}^{r}=0$ ).

Consider for a moment what happens to these pole/zero locations as we start to move $\delta_{1}^{r}, \delta_{2}^{r}$ away from zero. If we restrict $\delta_{1}^{r}, \delta_{2}^{r} \in \mathbb{R}$ then we get the motion indicated by the arrows in figure 10 , so that initially the poles stay in the left half-plane and the zeros stay in the right half-plane. However if we allow $\delta_{1}^{r}, \delta_{2}^{r} \in \mathbb{C}$ then we get motion inside the indicated circles. In this case we find that the poles and zeros can cross the imaginary axis for much smaller values of the uncertainty. Thus we would expect to see a fairly large gap between mixed and complex $\mu$ for this type of problem.

A complex $\mu$ synthesis controller was designed for this problem and the complex and mixed $\mu$ plots are shown in the right-hand plot of figure 11. Once again only the upper bounds are shown. This controller achieves a peak value for complex $\mu$ of about 2.6 , with mixed $\mu$ being about the same. The results for the mixed $\mu$ synthesis controller are shown in the left-hand plot of figure 11. This controller does not greatly affect the peak value of complex $\mu$, but reduces the peak value of mixed $\mu$ to about 1.1. Thus we do indeed find that there is a large gap between mixed and complex $\mu$ for this problem, and the mixed $\mu$ synthesis procedure is able to exploit that fact to significantly improve on the complex $\mu$ synthesis controller. 


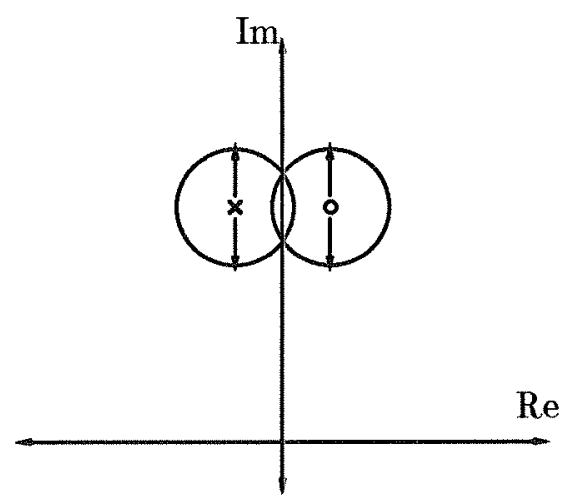

Figure 10: Pole/zero motion for the modal example
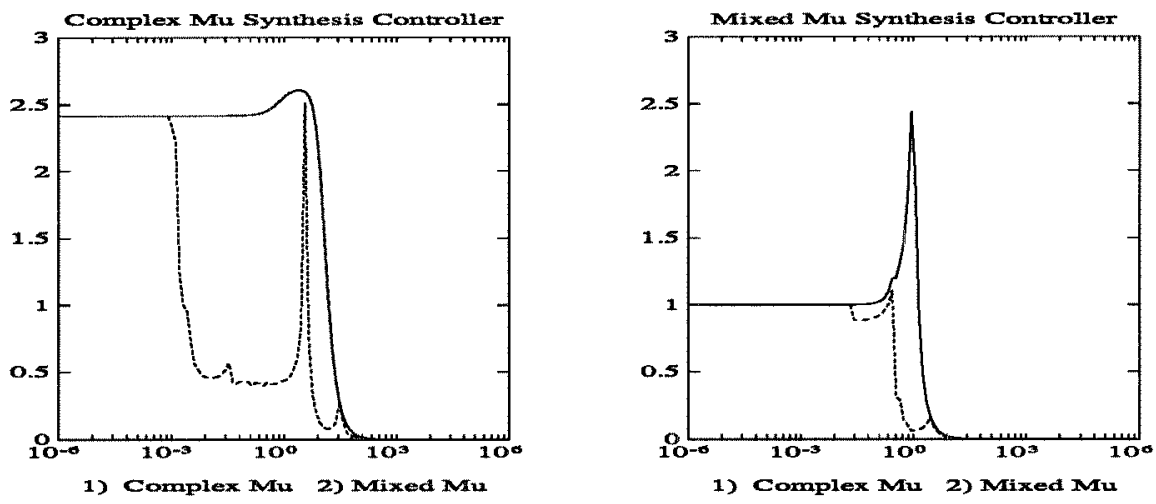

Figure 11: Complex and mixed $\mu$ plots for the modal example

Of course the two designs presented here are only for simple example problems, where the controller is chosen to optimize the robust stability margin with respect to some uncertain real parameters. A practical control design for a real problem involves a great many other issues. In particular one would need to select weights so as to reflect a more suitable performance criterion, rather than merely optimizing the stability margin at the expense of everything else (see [Y $\AA$ ]). Nevertheless these examples do illustrate the workings of the new method, and the initial results are encouraging. A further example may be found in [Y $\AA]$, where this design technique is compared to classical methods for a robust performance problem with real parameter uncertainty.

\section{Conclusion}

The general mixed $\mu$ synthesis problem has been considered, and a " $D, G-K$ iteration" procedure developed to tackle the problem. This procedure relies heavily on the theoretical properties of $\mu$ and its bounds, and has guaranteed convergence to a local minimum of the problem (which is nonconvex). This yields a stabilizing controller which attempts to minimize the peak value across frequency of mixed $\mu$, and hence provide the best possible robust performance in the face of mixed 
(real and complex) uncertainties. The controller synthesis procedure is applicable to general mixed $\mu$ robust performance problems, although further work is required to develop a practical solution to the repeated parameter case. This procedure has been implemented in software and applied to a number of example problems involving real parametric uncertainty, where it was able to synthesize a controller yielding a lower value for mixed $\mu$ (and hence better robust performance) than could be obtained with standard complex $\mu$ synthesis techniques.

\section{Acknowledgements}

I would like to thank John Doyle, Andy Packard and Matt Newlin for numerous helpful discussions. I would also like to thank Gary Balas and Keith Glover for lending me some of their expertise, and their software. This work was supported by ONR, NSF, NASA, and Rockwell International.

\section{References}

[BDG+91] Balas, G., Doyle, J., Glover, K., Packard, A., And Smith, R. The $\mu$ analysis and synthesis toolbox. MathWorks and MUSYN, 1991.

[BCD89] Balas, G. J., Chu, C., And Doyle, J. C. Vibration damping and robust control of the JPL/AFAL experiment using $\mu$-synthesis. In Proceedings of the $28^{\text {th }}$ Conference on Decision and Control (1989), pp. 2689-2694.

[BD90] Balas, G. J., AND Doyle, J. C. Robustness and performance tradeoffs in control design for flexible structures. In Proceedings of the $29^{\text {th }}$ Conference on Decision and Control (1990), pp. 2999-3010.

[BPD +91$]$ Balas, G. J., Packard, A., Doyle, J. C., Glover, K., and Smith, R. Development of advanced control design software for researchers and engineers. In Proceedings of the American Control Conference (1991), pp. 996-1001.

[BYD92] Balas, G. J., Young, P. M., and Doyle, J. C. $\mu$ based control design as applied to a large space structure: Control design for the minimast facility. NASA CSI/GI final report, June 1992.

[BYDM] Braftz, R. D., Young, P. M., Doyle, J. C., and Morari, M. Computational complexity of $\mu$ calculation. to appear in IEEE Transactions on Automatic Control.

[Doy82] Doyle, J. Analysis of feedback systems with structured uncertainty. IEE Proceedings, Part D 129, 6 (Nov. 1982), 242-250.

[DGKF89] Doyle, J., Glover, K., Khargonekar, P., and Francis, B. State space solutions to $\mathcal{H}_{2}$ and $\mathcal{H}_{\infty}$ control problems. IEEE Transactions on Automatic Control 34, 8 (August 1989), 831-847.

[Doy92] Doyle, J. C. Private Communication, 1992.

[Doy85] Doyle, J. C. Structured uncertainty in control system design. In Proceedings of the $24^{\text {th }}$ Conference on Decision and Control (1985), IEEE, pp. 260-265. 
[DFT92] Doyle, J. C., Francis, B. A., and Tannenbaum, A. R. Feedback Control Theory. Macmillan, New York, 1992.

[FTD91] Fan, M. K. H., Tits, A. L., and Doyle, J. C. Robustness in the presence of mixed parametric uncertainty and unmodeled dynamics. IEEE Transactions on Automatic Control AC-36 (1991), 25-38.

[Fra87] Francis, B. A. A Course in $\mathcal{H}_{\infty}$ Control Theory. Springer Verlag, New York, 1987.

[LS88] Little, J. N., And Shure, L. The signal processing toolbox. MathWorks 1988.

[OS75] Oppenheim, A. V., and Schaffer, R. W. Digital Signal Processing. Prentice Hall, New Jersey, 1975.

[PD93] Packard, A. K., And Doyle, J. C. The complex structured singular value. Automatica 29 (1993), 71-109.

[RM92] Rantzer, A., And Megretsky, A. A convex parameterization of robustly stabilizing controllers. Tech. rep., Department of Mathematics, Royal Institute of Technology, Stockholm, Sweden, 1992.

[RP] RohN, J., AND PolJaK, S. Checking robust nonsingularity is NP-hard. to appear in Mathematics of Control, Signals and Systems.

[SD91] Stein, G., And Doyle, J. C. Beyond singular values and loop shapes. Journal of Guidance, Control and Dynamics 14, 1 (January 1991), 5-16.

[You93] Young, P. M. Robustness with Parametric and Dynamic Uncertainty. PhD thesis, California Institute of Technology, 1993.

[YÅ] Young, P. M., And Åström, K. J. $\mu$ meets Bode. Manuscript in Preparation.

[YND91] Young, P. M., Newlin, M. P., And Doyle, J. C. $\mu$ analysis with real parametric uncertainty. In Proceedings of the $30^{t h}$ Conference on Decision and Control (1991), IEEE, pp. 1251-1256.

[YND92] Young, P. M., Newlin, M. P., And Doyle, J. C. Practical computation of the mixed $\mu$ problem. In Proceedings of the American Control Conference (1992), pp. 21902194.

[ZDG] Zhou, K., Doyle, J., And Glover, K. Robust and optimal control. Book in Preparation. 